\title{
Probabilistic model for the simulation of secondary electron emission
}

\author{
M. A. Furman* and M. T. F. Pivi ${ }^{\dagger}$ \\ Center for Beam Physics, Accelerator and Fusion Research Division, Lawrence Berkeley National Laboratory, \\ MS 71-259 Berkeley, California 94720
}

(Received 29 July 2002; published 31 December 2002)

\begin{abstract}
We provide a detailed description of a model and its computational algorithm for the secondary electron emission process. The model is based on a broad phenomenological fit to data for the secondary-emission yield and the emitted-energy spectrum. We provide two sets of values for the parameters by fitting our model to two particular data sets, one for copper and the other one for stainless steel.
\end{abstract}

DOI: 10.1103/PhysRevSTAB.5.124404

\section{INTRODUCTION}

The existence of the electron-cloud effect (ECE) [1,2], whose first and most prominent manifestation is beaminduced multipacting [3,4], has been firmly established experimentally at several storage rings [5-8]. Generally speaking, the ECE is a consequence of the strong coupling between a charged-particle beam and the vacuum chamber that contains it via a cloud of electrons in the chamber. The ECE is detrimental to the performance of modern storage rings, which typically make use of intense beams, closely spaced bunches, and/or vacuum chambers of small transverse dimensions.

For the past several years we have been studying the ECE by means of multiparticle simulations with a code that includes a detailed probabilistic model of the secondary-emission process $[9,10]$, which is one of the critical contributors to the ECE. The input ingredients of the model are the secondary-emission yield (SEY) $\delta$ and the emitted-energy spectrum of the secondary electrons $d \delta / d E$. The main result from our construction is the set of probabilities for the generation of electrons. This set of probabilities is embodied in a Monte Carlo procedure that generates simulated secondary-emission events given the primary electron energy and angle. We represent $\delta$ and $d \delta / d E$ by fairly general phenomenological fits constructed to obtain good agreement with a broad range of data. An additional virtue of the model is that it is mathematically self-consistent; by this we mean that the event generator is constructed so that (1) when averaging over an infinite number of secondary-emission events, the reconstructed $\delta$ and $d \delta / d E$ are guaranteed to agree with the corresponding input quantities; (2) the energy integral of $d \delta / d E$ is guaranteed to equal $\delta$; (3) the energy of any given emitted electron is guaranteed not to exceed the primary energy; and (4) the aggregate energy of the electrons emitted in any multielectron event is also guaranteed not to exceed the primary energy. The main challenge is the construction of the joint probability distributions for events in which two or more secondary electrons are generated in such a way as to satisfy these constraints.
PACS numbers: 79.20.Hx, 34.80.Bm, 29.27.Bd, 02.70.Uu

The main purpose of this article is the description of the model and its computer implementation in the larger ECE simulation code. We also provide sample fits to existing data on the SEY and emitted-energy spectrum. With regards to this latter quantity, we provide here a correction to a previously used [9] expression that was conceptually incorrect. Although the emitted-energy spectrum is not computed nor used directly in the ECE simulation code, it is used to extract parameters from the data which are then fed as input to the simulation. Recent work has shown, in some cases, an unexpectedly strong sensitivity of the overall simulation results on low-energy details of the SEY and the energy spectrum [11,12] that remains to be fully characterized and understood. Motivated by this, we have paid particular attention to the above-mentioned low-energy details in our model. Therefore, although the model involves a fair number of adjustable parameters, and some of them cannot be uniquely pinned down by presently available data, its mathematical consistency and its good overall agreement with secondary-emission data ensure that the abovementioned sensitivity cannot be attributed to mathematical artifacts of the model nor to inadequate representation of the data.

The Monte Carlo technique has been used before for the description of the secondary-emission process. In a more traditional approach, the main ingredient is a microscopic model for the secondary-emission material, typically specified by the electron and ion distributions, and by the elastic and inelastic cross sections for the collision of the primary and secondary electrons with the ions and with the other electrons in the material [13]. In this approach, one may infer microscopic properties of the material by comparing measured data for $\delta$ and $d \delta / d E$ with the corresponding quantities computed from the model. On the other hand, in the approach we present in this article, as mentioned above, the main ingredient is the measured data for $\delta$ and $d \delta / d E$, and the main result is the set of joint probability functions for the emission of secondary electrons. Thus our model is essentially phenomenological and does not afford a direct insight into 
the properties of the material or the theory of secondary emission. In particular, some of our fitting formulas are of a different form from those based on the theory of metals. However, although our formula for the true-secondary yield (Sec. IIID1) is different from the conventional one [14], it does incorporate the well-established rangeenergy relation [15].

In Sec. II we describe the secondary-emission process by first briefly recapitulating the basic phenomenology and then providing the probabilistic description of the emission process in terms of the "most differential probabilities" $\mathbb{P}_{n}$, which constitute the basic building blocks for our model. This probabilistic description is quite general, and we believe its validity to be rooted in general principles of the quantum theory for the secondaryemission process. In Sec. IIC we define a specific phenomenological model for the $\mathbb{P}_{n}$ 's by following the principle of maximum simplicity consistent with the data. In particular, we strictly enforce the condition that the energy of any secondary electron may not exceed that of the incident (primary) electron, a fact that is clearly exhibited by secondary energy spectrum data. In addition, we also impose the same restriction on the aggregate secondary energy. Although we are not aware of experimental data supporting this latter restriction, we believe it to be true on account of general physical principles. In Sec. III we continue the definition of our model by providing detailed parametrizations for each of the three components of the SEY based on various reviews of the theory and phenomenology of the subject [14,16-18]. In Sec. IV we carry out the analytic calculation of the energy spectrum within our model. In Sec. $\mathrm{V}$ we provide the algorithmic description of the probabilisitic model just constructed, as implemented in our ECE simulation code. In Sec. VI we use the energy spectrum, along with the three components of the SEY, to fit the data and extract the various parameters of the model. In Sec. VII we summarize our conclusions. The various Appendices provide (i) mathematical details of the analytic calculation of the energy spectrum. (ii) A simplified alternative model for the $\mathbb{P}_{n}$ 's that does not respect the abovementioned constraints on the secondary energy, but offers a simplified calculation of the spectra that is approximately valid for high enough primary energy. (iii) Definition of the "jet" energy spectrum. (iv) The Monte Carlo method of computing the energy spectra. (v) Alternative parametrizations of aspects of the SEY.

\section{MODEL OF SECONDARY ELECTRON EMISSION}

\section{A. Basic phenomenology}

The two main quantities used in the experimental study of the secondary-emission process are the SEY $\delta$ and the emitted-energy spectrum $d \delta / d E[17,18]$. To define these, we consider a steady monoenergetic electron beam im- pinging on a surface. The SEY is defined by

$$
\delta=\frac{I_{s}}{I_{0}}
$$

where $I_{0}$ is the incident electron beam current and $I_{s}$ is the secondary current, i.e., the electron current emitted from the surface. The yield is a function of the kinetic energy $E_{0}$ of the incident electron beam, its incident angle $\theta_{0}$, and the type of surface material and its state of conditioning. ${ }^{1}$ For applications to the ECE, we are primarily interested in incident energies $E_{0}$ below a few keV's, although the framework presented here is formally valid for all energies.

By applying a retarding voltage $V$ in front of the secondary current detector one can select those electrons that are emitted with individual energies $E_{k} \geq E=e V$. The cumulative emitted-energy spectrum $S\left(E_{0}, E\right)$ is then defined to be

$$
S\left(E_{0}, E\right)=\frac{I_{s}(E)}{I_{0}},
$$

where $I_{s}(E)$ is the secondary current that overcomes the retarding voltage (for notational conciseness we suppress a dependence of $S$ on $\theta_{0}$ ). The emitted-energy spectrum $d \delta / d E$ is defined to be

$$
\frac{d \delta}{d E}=-\frac{\partial S\left(E_{0}, E\right)}{\partial E}
$$

where the minus sign ensures that $d \delta / d E>0$ (the emitted-energy spectrum $d \delta / d E$ can also be measured directly by means of a magnetostatic or electrostatic energy analyzer $[19,20])$. Note that $I_{s}(0)$ in Eq. (2) is what is simply called $I_{s}$ in Eq. (1), so that $S\left(E_{0}, 0\right)=$ $\delta\left(E_{0}\right)$, hence

$$
\int_{0}^{\infty} d E \frac{d \delta}{d E}=\delta\left(E_{0}\right)
$$

For more detailed descriptions of the secondaryemission process one may require additional variables or measured quantities. For example, if the surface has an anisotropy defined by a preferred direction such as a crystal axis or grooves arising from the fabrication process [21], one may need to specify an azimuthal incident angle $\phi_{0}$ in addition to the polar angle $\theta_{0}$. If the secondary electron detector is capable of detecting the emitted-angle dependence of the secondary electrons, an appropriate emission-angle dependence should be incorporated into $S\left(E_{0}, E\right)$ [17]. If the incident electron beam is polarized, one may need to define the yield and emitted-energy spectrum separately for each spin polarization state. In this article, however, we are not concerned with such additional details: we consider only

\footnotetext{
${ }^{1}$ We adopt the convention that $\theta_{0}$ is measured relative to the normal of the surface.
} 
homogeneous materials, unpolarized incident electrons, and undetected (hence averaged over) polarizations of the emitted electrons. In addition, we wholly neglect the contribution of Auger electrons [22] to $d \delta / d E$, as well as the "characteristic energy loss" mechanism [18], as these effects are expected to contribute negligibly to the electron-cloud effect.

\section{B. Probabilistic description}

We now provide a microscopic, i.e., event-by-event, description of the secondary-emission process, where an "event" is a single electron-surface collision. This process is quantum mechanical hence probabilistic in nature; thus an electron with kinetic energy $E_{0}$ striking a surface at an angle $\theta_{0}$ will yield $n$ secondary electrons with a probability $P_{n}\left(E_{0}, \theta_{0}\right), n=1, \ldots, \infty$, as sketched in Fig. 1. The $P_{n}$ 's obviously satisfy

$$
\sum_{n=0}^{\infty} P_{n}=1, \quad P_{n} \geq 0
$$

where $P_{0}$ is the probability that the incident electron is absorbed without emission. In terms of the $P_{n}$ 's, the SEY defined in Sec. IIA is simply the average electron multiplicity in the collision,

$$
\delta=\langle n\rangle=\sum_{n=1}^{\infty} n P_{n}
$$

Although much is known experimentally about the energy spectrum and angular distribution of the secondary electrons, the knowledge of $\delta, d \delta / d E$, and the $P_{n}$ 's is not enough for an event-by-event simulation of the secondary-emission process. Such a simulation requires the knowledge of the "most differential probability"

$$
\mathbb{P}_{n}=\frac{d P_{n}}{d E_{1} d \Omega_{1} d E_{2} d \Omega_{2} \cdots d E_{n} d \Omega_{n}}
$$

for $1 \leq n<\infty$, i.e., the joint probability for the $n$ electrons in the final state to be emitted with kinetic energies $E_{1}, E_{2}, \ldots, E_{n}$ into the solid angles $\Omega_{1}=\left(\theta_{1}, \phi_{1}\right), \Omega_{2}=$ $\left(\theta_{2}, \phi_{2}\right), \ldots, \Omega_{n}=\left(\theta_{n}, \phi_{n}\right)$ when an electron strikes the surface at a given energy $E_{0}$ and angle $\theta_{0}$.

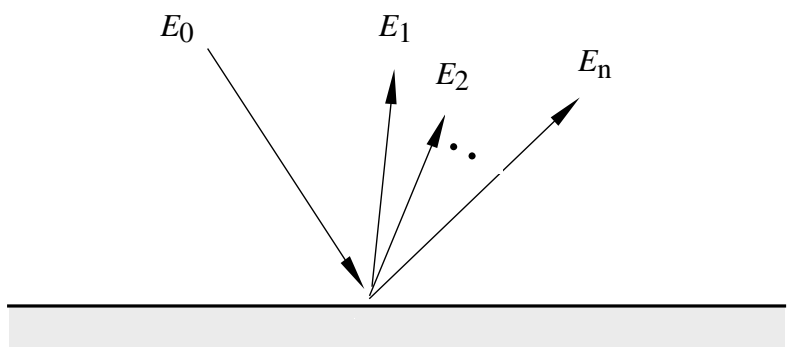

FIG. 1. A single electron with energy $E_{0}$ strikes a surface yielding $n$ secondary electrons with energies $E_{1}, E_{2}, \ldots, E_{n}$.
The phase-space probabilities $\mathbb{P}_{n}$ contain all the information relevant to the secondary-emission process. They correspond to the transition probabilities $\left|S_{f i}\right|^{2}$, where $S_{f i}$ is the quantum transition amplitude from an initial state $i$ to a final state $f$. In our case the initial state $i$ represents the incident electron, characterized by its energy $E_{0}$ and angle $\theta_{0}$, while the final state $f$ represents the $n$ emitted electrons, and is characterized by their energies and emission directions. The $\mathbb{P}_{n}$ 's are in principle calculable from the quantum theory of the surface material and in principle measurable. We are not aware, however, of any such calculations or measurements, which must surely be very challenging. The construction of a phenomenological model for the $\mathbb{P}_{n}$ 's is the central goal of this article. Although such a construction is not unique, we shall be guided by the principle of maximum simplicity consistent with available data for $\delta$ and $d \delta / d E$.

If we define the $n$-body volumes of kinetic energy and solid angle, respectively, as $(d E)_{n} \equiv d E_{1} d E_{2} \cdots d E_{n}$ and $(d \Omega)_{n} \equiv d \Omega_{1} d \Omega_{2} \cdots d \Omega_{n}$, then $\mathbb{P}_{n}=d P_{n} /(d E)_{n}(d \Omega)_{n}$. The probability $P_{n}$ is obtained by integrating $\mathbb{P}_{n}$ over the entire phase space of the secondary electrons,

$$
P_{n}=\int(d E)_{n}(d \Omega)_{n} \mathbb{P}_{n}, \quad n \geq 1,
$$

and the absorption probability is then given by

$$
P_{0}=1-\sum_{n=1}^{\infty} P_{n}
$$

(again, we suppress a dependence of the $P_{n}$ 's on $E_{0}$ and $\theta_{0}$ for notational clarity).

The cumulative secondary energy spectrum $S\left(E_{0}, E\right)$ is then given by

$$
S\left(E_{0}, E\right)=\sum_{n=1}^{\infty} \int(d E)_{n}(d \Omega)_{n} \mathbb{P}_{n} \sum_{k=1}^{n} \theta\left(E_{k}-E\right),
$$

where the $\theta$ functions ensure that only those electrons emitted with an energy $\geq E$ are counted, and that the count is precisely equal to the number of such electrons. Finally, Eq. (3) yields ${ }^{2}$

$$
\frac{d \delta}{d E}=\sum_{n=1}^{\infty} \int(d E)_{n}(d \Omega)_{n} \mathbb{P}_{n} \sum_{k=1}^{n} \delta\left(E_{k}-E\right) .
$$

\section{Model for $\mathbb{P}_{\boldsymbol{n}}$ \\ 1. Emission angles}

For simplicity we assume that $\mathbb{P}_{n}$ is of the form

$$
\mathbb{P}_{n}=A_{n}\left(\Omega_{1}, \ldots, \Omega_{n}\right) \times \frac{d P_{n}}{(d E)_{n}},
$$

${ }^{2}$ The Dirac delta functions appearing in the sum over $k$ in Eq. (11) should not be confused with the SEY. 
where $\Omega_{k}=\left(\theta_{k}, \phi_{k}\right)$ is the emission direction of the $k$ th secondary electron, and $d P_{n} /(d E)_{n}$ does not depend on $\Omega_{k}$. This formula implies that the emission energy is uncorrelated with the emission angle. Experimentally, it is known [17] that the true-secondary electrons have a $\sim \cos \theta$ distribution in angle, which is fairly independent of the primary incident angle $\theta_{0}$ and incident energy $E_{0}$. This is not quite true of the elastically reflected and rediffused electrons (see below), which have a more complicated angular distribution. Nevertheless, following the simplicity principle, we assume the same emission-angle distribution for all electrons, regardless of the physical mechanism by which they were generated. Thus we assume

$$
A_{n}\left(\Omega_{1}, \ldots, \Omega_{n}\right)=\left(\frac{\alpha+1}{2 \pi}\right)^{n} \prod_{k=1}^{n} \cos ^{\alpha} \theta_{k},
$$

where $\alpha$ is an adjustable parameter expected to be close to 1. This form for $A_{n}$ also implies that the emission angles are fully uncorrelated from each other (the azimuthal emission angle $\theta_{k}$ is defined relative to the normal to the surface at the point where the primary electron strikes). The normalization is such that

$$
\int(d \Omega)_{n} A_{n}\left(\Omega_{1}, \ldots, \Omega_{n}\right)=1,
$$

so that the energy part of the distribution is given by

$$
\frac{d P_{n}}{(d E)_{n}}=\int(d \Omega)_{n} \mathbb{P}_{n}
$$

The above normalization for $A_{n}$ and Eq. (8) imply that $d P_{n} /(d E)_{n}$ satisfies

$$
\int(d E)_{n} \frac{d P_{n}}{(d E)_{n}}=P_{n}
$$

\section{Emission energies}

We now make the assumption that $d P_{n} /(d E)_{n}$ is of the form

$$
\frac{d P_{n}}{(d E)_{n}}=\theta\left(E_{0}-\sum_{k=1}^{n} E_{k}\right) \prod_{k=1}^{n} f_{n}\left(E_{k}\right) \theta\left(E_{k}\right) \theta\left(E_{0}-E_{k}\right),
$$

where $f_{n}\left(E_{k}\right)$ is the energy distribution of the $k$ th emitted electron in an event with a total number $n$ of secondary electrons. The $\theta$ function in front ensures that the aggregate energy of the emitted electrons does not exceed the primary electron energy. Physically, this constraint means that the secondary electrons are emitted in an almost uncorrelated fashion: they "know" about each other just enough that they will not extract energy from the surface material. The functions $\theta\left(E_{0}-E_{k}\right)$ ensure that the energy of any given emitted electron does not exceed the primary electron energy either, a fact that is well supported by experimental data on the emittedenergy spectrum.

In Appendix $\mathrm{C}$ we consider, as a simplified alternative, a fully uncorrelated model defined by an expression for $d P_{n} /(d E)_{n}$ similar to (17) without the $\theta$ functions [Eq. (C1)]. This model has the advantage of simplicity over (17), but if suffers from the deficiency that the energy of any given secondary electron has a nonzero probability to exceed $E_{0}$, contrary to experimental results. Nevertheless, if the functions $f_{n}(E)$ 's vanish at large $E$, the $\theta$ function in (17) may be effectively neglected for large $E_{0}$, hence the results obtained from (17) must coincide with those from $(\mathrm{C} 1)$ in this limit.

Equation (16) implies

$$
\int_{0}^{E_{0}} \prod_{k=1}^{n}\left\{d E_{k} f_{n}\left(E_{k}\right)\right\} \theta\left(E_{0}-\sum_{k=1}^{n} E_{k}\right)=P_{n}\left(E_{0}\right)
$$

which we will later use to normalize the $f_{n}$ 's to the $P_{n}$ 's. Note that Eq. (18) implies the sum rule

$$
\int_{0}^{E_{0}} d E f_{n}(E) P_{n-1}\left(E_{0}-E\right)=P_{n}\left(E_{0}\right)
$$

where the term $P_{0}$ appearing in the integrand for the choice $n=1$ must be interpreted, not as the absorption probability (9), but rather as $\theta\left(E_{0}-E\right)$.

\section{THE THREE COMPONENTS OF THE SEY}

\section{A. The basic assumption}

The conventional picture of secondary emission, which we base on various reviews of the subject [14,16-18], can be summarized as follows: when a steady current $I_{0}$ of electrons impinges on a surface, a certain portion $I_{e}$ is backscattered elastically while the rest penetrates into the material. Some of these electrons scatter from one or more atoms inside the material and are reflected back out. These are the so-called "rediffused" electrons, and we call the corresponding current $I_{r}$. The rest of the electrons interact in a more complicated way with the material and yield the so-called "true-secondary electrons," whose current we call $I_{t s}$. The yields for each type of electron are defined by $\delta_{e}=I_{e} / I_{0}, \delta_{r}=I_{r} / I_{0}$, and $\delta_{t s}=I_{t s} / I_{0}$, so that the total SEY is

$$
\begin{aligned}
\delta & =\left(I_{e}+I_{r}+I_{t s}\right) / I_{0} \\
& =\delta_{e}+\delta_{r}+\delta_{t s} \\
& =P_{1}+2 P_{2}+3 P_{3}+\cdots,
\end{aligned}
$$

where (20c) follows from (6). There is no fundamental distinction between the backscattered and rediffused mechanisms, and we treat them on an equal footing in our model. However, we have found it advantageous, for the purposes of phenomenological fits, to separate them into two components. At the quantum level, of course, the 
distinction between the three types of electrons is unphysical. Nevertheless, in practice there exists a conventional criterion $[17,18]$, which we follow, that leads to this distinction, and hence to the separate measurements of $\delta_{e}, \delta_{r}$, and $\delta_{t s}$. The criterion is based on the three main regions, in secondary energy $E$, exhibited by $d \delta / d E$. As an example, Fig. 2 shows the contributions of the three components for the case of an incident electron beam of energy $E_{0}=300 \mathrm{eV}$ impinging on a stainless steel surface.

In order to assign the three components of $\delta$ to the $P_{n}$ 's, we now make a simplifying assumption; namely, the elastic and rediffused electrons are only produced in one-electron events $(n=1)$, while the true-secondary electrons are produced in events with any number of secondary electrons $(n \geq 1)$. Therefore, in our model, this assumption means that in any given event backscattered and rediffused electrons are never accompanied by true secondaries, and conversely, when two or more truesecondary electrons are produced, they are never accompanied by either backscattered or rediffused electrons. This assumption of mutual exclusion appears to be consistent with the experimental data on the emitted-energy spectrum for incident energies above $\sim 50 \mathrm{eV}$; however, it is not required by the data, and we have no good arguments for it in general. We adopt it here for all energies as it offers perhaps the simplest (but not the only) mathematically consistent characterization of the secondary-emission process. The basic picture is sketched in Fig. 3.

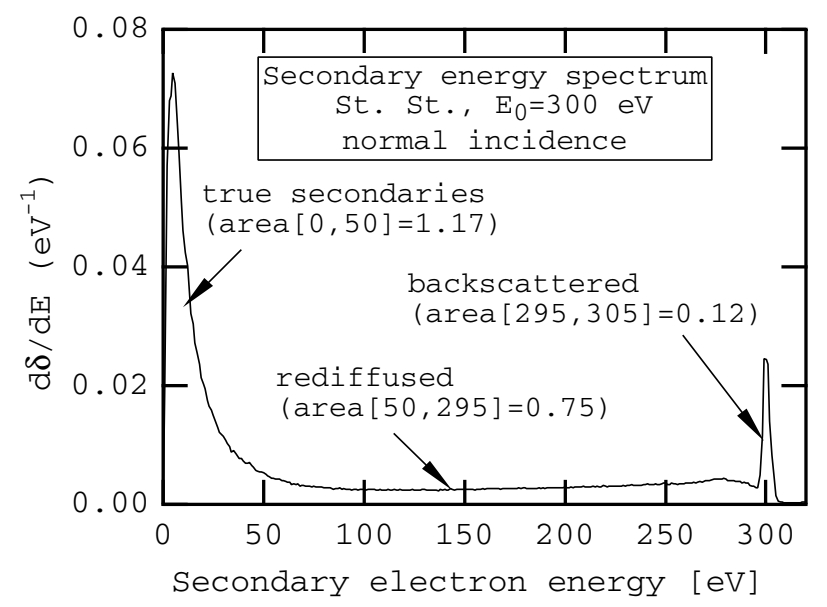

FIG. 2. A sample of the measured energy spectrum $d \delta / d E$ for an unconditioned sample of stainless steel at $E_{0}=300 \mathrm{eV}$, normal incidence. The three components of the secondary yield are given by the values of "area $\left[E_{1}, E_{2}\right]$," each of which represents the integrated spectrum between $E_{1}$ and $E_{2}$. Thus for this case, $\delta_{t s}=1.17, \delta_{r}=0.75$, and $\delta_{e}=0.12$, for a total SEY $\delta=2.04$. The upper energy cutoff for the true secondaries is somewhat arbitrarily, but conventionally, chosen to be $50 \mathrm{eV}$. Data courtesy of R. Kirby.

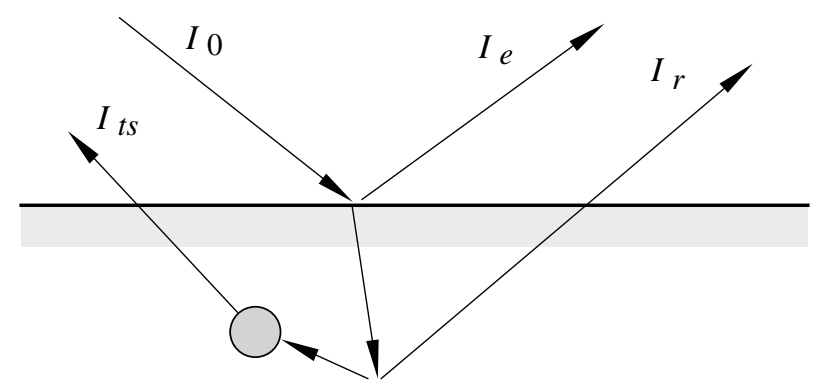

FIG. 3. Sketch of the currents that are used to define the different components of secondary emission. The blob is meant to emphasize a nontrivial interaction yielding true-secondary electrons. This sketch embodies a mutual-exclusion property of the three types of emission processes, spelled out in the text.

In terms of the $P_{n}$ 's, this assumption implies

$$
\begin{aligned}
& P_{1}=P_{1, e}+P_{1, r}+P_{1, t s}, \\
& P_{n}=P_{n, t s}, \quad n \geq 2,
\end{aligned}
$$

which implies an absorption probability

$$
P_{0}=1-\sum_{n=1}^{\infty} P_{n}=1-P_{1, e}-P_{1, r}-\sum_{n=1}^{\infty} P_{n, t s}
$$

and also

$$
\begin{aligned}
\delta_{e} & =P_{1, e}, \\
\delta_{r} & =P_{1, r}, \\
\delta_{t s} & =\sum_{n=1}^{\infty} n P_{n, t s} .
\end{aligned}
$$

In terms of the $f_{n}$ 's, we have

$$
\begin{aligned}
& f_{1}=f_{1, e}+f_{1, r}+f_{1, t s}, \\
& f_{n}=f_{n, t s}, \quad n \geq 2,
\end{aligned}
$$

where the functions $f_{1, e}, f_{1, r}, f_{n, t s}, P_{1, e}, P_{1, r}$, and $P_{n, t s}$ remain to be specified.

Should more detailed data on secondary emission invalidate the mutual-exclusion assumption, Eqs. (21), (23), and (24) would have to be modified accordingly. Note that our construction does not guarantee that $\delta_{e}+\delta_{r} \leq 1$, a condition that must be satisfied in our model. This condition must be enforced by appropriate parameter choices, although this is rarely a problem in practice. Note also that there is no safeguard that prevents $P_{1}$ from exceeding unity nor $P_{0}$ from becoming negative. These conditions must also be enforced by appropriate parameter choices, but this might be problematic in certain cases of practical interest. A construction of the $P_{n}$ 's that does guarantee $P_{1} \leq 1$ and $P_{0} \geq 0$ is presented in Sec. IIID2 below. 


\section{B. Model for backscattered electrons}

Experimental data $[17,18]$ suggests that a sensible form for $\delta_{e}\left(E_{0}, \theta_{0}\right)$ at normal incidence $\left(\theta_{0}=0\right)$ might be given by

$$
\delta_{e}\left(E_{0}, 0\right)=P_{1, e}(\infty)+\left[\hat{P}_{1, e}-P_{1, e}(\infty)\right] e^{-\left(\left|E_{0}-\hat{E}_{e}\right| / W\right)^{p} / p}
$$

This function peaks at an energy $E_{0}=\hat{E}_{e}$ provided $\hat{P}_{1, e}>P_{1, e}(\infty)$, which we assume to be the case. For the energy probability function $f_{1, e}$ we assume a form that roughly matches the elastic component of the spectrum $d \delta / d E$ (Figs. 5 and 7), namely,

$$
f_{1, e}=\theta(E) \theta\left(E_{0}-E\right) \delta_{e}\left(E_{0}, \theta_{0}\right) \frac{2 e^{-\left(E-E_{0}\right)^{2} / 2 \sigma_{e}^{2}}}{\sqrt{2 \pi} \sigma_{e} \operatorname{erf}\left(E_{0} / \sqrt{2} \sigma_{e}\right)}
$$

which is normalized so that it satisfies

$$
\int_{0}^{E_{0}} d E f_{1, e}(E)=\delta_{e}\left(E_{0}\right)
$$

The fact that $\delta_{e} \neq 0$ for $E_{0}=0$ implies that expression (26) for $f_{1, e}$ diverges as $E_{0} \rightarrow 0$. This divergence is not unphysical, however, because it is integrable, viz., Eq. (27).

\section{Model for the rediffused electrons}

Experimental data $[17,18]$ suggest that a sensible form for $\delta_{r}$ at normal incidence $\left(\theta_{0}=0\right)$ might be given by

$$
\delta_{r}\left(E_{0}, 0\right)=P_{1, r}(\infty)\left[1-e^{-\left(E_{0} / E_{r}\right)^{r}}\right] .
$$

For $f_{1, r}$ we assume

$$
f_{1, r}=\theta(E) \theta\left(E_{0}-E\right) \delta_{r}\left(E_{0}, \theta_{0}\right) \frac{(q+1) E^{q}}{E_{0}^{q+1}}
$$

which satisfies the normalization condition

$$
\int_{0}^{E_{0}} d E f_{1, r}(E)=\delta_{r}\left(E_{0}\right)
$$

\section{Model for the true-secondary electrons}

\section{Yield and energy distribution function}

The energy and angular dependence of $\delta_{t s}$ are well fit experimentally $[14,16,17]$ by an approximately universal [23] scaling function $D(x)$ such that

$$
\delta_{t s}\left(E_{0}, \theta_{0}\right)=\hat{\delta}\left(\theta_{0}\right) D\left[E_{0} / \hat{E}\left(\theta_{0}\right)\right],
$$

so that all dependence on the surface and incident angle is contained in $\hat{\delta}$ and $\hat{E}$. The scaling function $D(x)$ is defined so that it satisfies the conditions $D(1)=1$ and $D^{\prime}(1)=0$, which are, of course, chosen to ensure that $\delta_{t s}$ reaches a peak value $\hat{\delta}$ at an energy $\hat{E}$. We have chosen the simplest form [9] for $D(x)$ that satisfies the abovementioned conditions and that allows good fits to the data $[21,24]$, namely,

$$
D(x)=\frac{s x}{s-1+x^{s}},
$$

where $s$ is an adjustable parameter that must be $>1$. In the literature one finds other parametrizations for $D(x)$, discussed in Appendix F. We have found, however, that some of these parametrizations do not fit actual data for technical surfaces as well as (32). We note that this formula implies $\delta_{t s} \sim E_{0}^{1-s}$ at large $E_{0}$, showing that it satisfies the conventional range-energy relation including scattering effects [15]. Further details are described in Appendix F.

As for the energy spectrum function we make the assumption

$$
f_{n, t s}=\theta(E) F_{n} E^{p_{n}-1} e^{-E / \epsilon_{n}},
$$

where $p_{n}$ and $\epsilon_{n}$ are phenomenological parameters (the finiteness of $\delta\left(E_{0}, \theta_{0}\right)$, however, demands $\left.p_{n}>0\right)$. Equations (18) and (A5) yield

$$
F_{n}^{n}=\frac{P_{n, t s}\left(E_{0}\right)}{\left[\epsilon_{n}^{p_{n}} \Gamma\left(p_{n}\right)\right]^{n} P\left(n p_{n}, E_{0} / \epsilon_{n}\right)},
$$

where $P(z, x)$ is the normalized incomplete gamma function (see Appendix A). The emission probability $P_{n, t s}$ is defined below in terms of $\delta_{t s}$; a dependence on the incident angle $\theta_{0}$ is contained in $P_{n, t s}$.

While Eq. (33) does not correspond to the expression derived from the theory of metals [16], it fits the data quite well (see Sec. VI), and allows many of the integrals to be carried out analytically, hence it is quite appropriate for our phenomenological approach.

\section{Emission probability}

a. Probability per incident electron. The final ingredient that must be defined in the model is the probability $P_{n, t s}$ for emitting $n$ true-secondary electrons, given $\delta_{t s}$, $\delta_{e}$, and $\delta_{r}$. Once we choose $P_{n, t s}$, the overall probabilities are given by Eqs. (21)-(23), namely,

$$
\begin{aligned}
& P_{0}=P_{0, t s}-\delta_{e}-\delta_{r}, \\
& P_{1}=P_{1, t s}+\delta_{e}+\delta_{r}, \\
& P_{n}=P_{n, t s}, \quad n \geq 2 .
\end{aligned}
$$

Since $P_{n, t s}$ satisfies the unitarity condition

$$
\sum_{n=0}^{\infty} P_{n, t s}=1
$$

so does the overall probability $P_{n}$. A simple choice for $P_{n, t s}$ is a Poisson distribution,

$$
P_{n, t s}=\frac{\delta_{t s}^{n}}{n !} e^{-\delta_{t s}, \quad 0 \leq n<\infty}
$$


which satisfies the requisite property $\langle n\rangle=\delta_{t s}$. Another possible choice is a binomial distribution,

$$
P_{n, t s}=\left(\begin{array}{c}
M \\
n
\end{array}\right) p^{n}(1-p)^{M-n}, \quad 0 \leq n \leq M,
$$

where $p=\langle n\rangle / M=\delta_{t s} / M$. This distribution limits the number of emitted secondary electrons to a maximum $M$. Although this limitation is not physical, it is in general quite innocuous for sufficiently large $M$, and it has the advantage that it leads to more controllable computations than the Poisson distribution. The parameter $p$ must be constrained to be $<1$ which implies, in turn, an upper limit on the acceptable value of $\delta_{t s}$. In practice we have found that $M=10$ gives sufficient accuracy for most simulations, and sets an upper limit $\delta_{t s}=10$, which is more than adequate for all practical materials.

b. Probability per penetrated electron. As mentioned in Sec. IIIA, it is clear from Eq. (35) that $P_{1}$ can exceed unity and $P_{0}$ can become negative even if $\delta_{e}$ and $\delta_{r}$ are constrained to satisfy $\delta_{e}+\delta_{r} \leq 1$. For example, these violations of basic probability properties can occur when $\delta_{t s} \gtrsim 1.2$ and $\delta_{e}+\delta_{r} \gtrsim 0.5$, a situation that can readily arise in practice. An alternative definition of the emission probabilities that guarantees $P_{0} \geq 0$ and $P_{1} \leq 1$ follows from considering the probabilities per unit penetrated electron current rather than per unit incident electron current. Referring to Fig. 3, it is clear that the current available for the production of true-secondary electrons is $I_{0}-I_{e}-I_{r}$. Thus the yield in terms of this penetrated current is

$$
\delta_{t s}^{\prime}=\frac{I_{t s}}{I_{0}-I_{e}-I_{r}}=\frac{\delta_{t s}}{1-\delta_{e}-\delta_{r}} .
$$

Although this definition is less practical from an experimental point of view, it allows a mathematically more consistent definition of the probabilities $P_{n}$, as we now show. We express the SEY component $\delta_{t s}^{\prime}$ as

$$
\delta_{t s}^{\prime}=\sum_{n=1}^{\infty} n P_{n, t s}^{\prime}
$$

where $P_{n, t s}^{\prime}$ is the probability, to be specified, for generating $n$ true-secondary electrons relative to the available penetrated current $I_{0}-I_{e}-I_{r}$. This probability must satisfy the unitarity condition

$$
\sum_{n=0}^{\infty} P_{n, t s}^{\prime}=1
$$

A comparison of Eqs. (23c) and (40) suggests the relation

$$
P_{n, t s}=\frac{\delta_{t s}}{\delta_{t s}^{\prime}} P_{n, t s}^{\prime}=\left(1-\delta_{e}-\delta_{r}\right) P_{n, t s}^{\prime}, \quad n \geq 1 .
$$

Although this relation is not implied by the comparison, we adopt it as the definition of the probability per incident electron $P_{n, t s}$. With this definition, Eqs. (21)-(23) determine the overall emission probabilities

$$
\begin{aligned}
& P_{0}=\left(1-\delta_{e}-\delta_{r}\right) P_{0, t s}^{\prime}, \\
& P_{1}=\left(1-\delta_{e}-\delta_{r}\right) P_{1, t s}^{\prime}+\delta_{e}+\delta_{r}, \\
& P_{n}=\left(1-\delta_{e}-\delta_{r}\right) P_{n, t s}^{\prime}, \quad n \geq 2,
\end{aligned}
$$

which should be compared with Eq. (35). It is easy to see that, if the condition $\delta_{e}+\delta_{r} \leq 1$ is satisfied, the above expressions do guarantee that $P_{1} \leq 1$ and $P_{0} \geq 0$, the equality holding only in the extreme case $\delta_{e}+\delta_{r}=1$. Note, however, that, in contrast with Eq. (36), the probabilities $P_{n, t s}$ defined in this way satisfy

$$
\sum_{n=0}^{\infty} P_{n, t s}=1-\delta_{e}-\delta_{r}
$$

which is, in general, $<1$ [we have extended Eq. (42) to $n=0]$. This lack of unitarity is as it should be: the deficit from unity in this sum represents precisely the fraction of incident electrons that yield backscattered and rediffused electrons, and hence is unavailable, according to our mutual-exclusion assumption, for true-secondary production.

As in the examples above, we may choose for $P_{n, t s}^{\prime}$ a Poisson distribution,

$$
P_{n, t s}^{\prime}=\frac{\delta_{t s}^{\prime n}}{n !} e^{-\delta_{t s}^{\prime}, \quad 0 \leq n<\infty,}
$$

which satisfies $\langle n\rangle=\delta_{t s}^{\prime}$, as it should in order for $\delta_{t s}^{\prime}$ to have the required meaning of being the average number of true-secondary electrons emitted per penetrated electron. We can also choose the binomial distribution,

$$
P_{n, t s}^{\prime}=\left(\begin{array}{c}
M \\
n
\end{array}\right) p^{n}(1-p)^{M-n}, \quad 0 \leq n \leq M,
$$

where $p=\langle n\rangle / M=\delta_{t s}^{\prime} / M$. Since $p$ must be $<1, M$ must be chosen $>\delta_{t s}^{\prime}$.

\section{E. Incident-angle dependence}

In our fits to data for vacuum chamber materials (noncrystalline metal with a rough surface) we have found that the incident-angle dependence of the total SEY is well fit by a multiplicative factor of the form $1+a_{1}(1-$ $\cos ^{a_{2}} \theta_{0}$ ) for incident angles in the range $0 \leq \theta_{0} \leq 84^{\circ}$ $[21,29]$. Not surprisingly, this dependence is much milder than the inverse power of $\cos \theta_{0}$ that is found in the literature for smooth surfaces $[16,26]$. For our purposes, we have assumed the same form for all three components of the SEY. Specifically, for the backscattered and rediffused components we set

$$
\begin{aligned}
& \delta_{e}\left(E_{0}, \theta_{0}\right)=\delta_{e}\left(E_{0}, 0\right)\left[1+e_{1}\left(1-\cos ^{e_{2}} \theta_{0}\right)\right], \\
& \delta_{r}\left(E_{0}, \theta_{0}\right)=\delta_{r}\left(E_{0}, 0\right)\left[1+r_{1}\left(1-\cos ^{r_{2}} \theta_{0}\right)\right],
\end{aligned}
$$

while for the true-secondary component we assume

$$
\begin{aligned}
& \hat{\delta}\left(\theta_{0}\right)=\hat{\delta}_{t s}\left[1+t_{1}\left(1-\cos ^{t_{2}} \theta_{0}\right)\right], \\
& \hat{E}\left(\theta_{0}\right)=\hat{E}_{t s}\left[1+t_{3}\left(1-\cos ^{t_{4}} \theta_{0}\right)\right] .
\end{aligned}
$$


A more complete discussion of other possible forms for the incident-angle dependence is presented in Appendix F.

\section{THE EMITTED-ENERGY SPECTRUM}

In order to extract more information from the data, we need a formula for the energy spectrum. From Eq. (11) and the model for $\mathbb{P}_{n}$ described in Sec. IIC we obtain

$$
\begin{aligned}
\frac{d \delta}{d E}= & \sum_{n=1}^{\infty} n f_{n}(E) \int_{0}^{\infty} \prod_{k=2}^{n}\left\{d E_{k} f_{n}\left(E_{k}\right)\right\} \\
& \times \theta\left(E_{0}-E-\sum_{k=2}^{n} E_{k}\right) \\
= & \sum_{n=1}^{\infty} n f_{n}(E) P_{n-1}\left(E_{0}-E\right)
\end{aligned}
$$

where the term $P_{0}\left(E_{0}-E\right)$ appearing in the $n=1$ term in (49b) must be interpreted not as the absorption probability but rather as $\theta\left(E_{0}-E\right)$. Using Eq. (19), it is clear from Eq. (49a) that $d \delta / d E$ obeys the sum rule

$$
\int_{0}^{E_{0}} d E \frac{d \delta}{d E}=\sum_{n=1}^{\infty} n P_{n}=\delta\left(E_{0}\right)
$$

as it should.

For the model described in Sec. III for the three components of the SEY we obtain

$$
\begin{aligned}
\frac{d \delta}{d E}=f_{1, e}+f_{1, r}+\frac{d \delta_{t s}}{d E}, & \begin{array}{c}
\text { at } E=(p-1) \epsilon . \\
\text { The cumulative spectrum can be similarly } \\
\text { Using Eqs. (10) and (17) we obtain }
\end{array} \\
S\left(E_{0}, E\right) & =\sum_{n=1}^{\infty} n \int_{E}^{E_{0}} d E_{1} f_{n}\left(E_{1}\right) \int_{0}^{\infty} d E_{2} \cdots d E_{n} f_{n}\left(E_{2}\right) \cdots f_{n}\left(E_{n}\right) \theta\left(E_{0}-\sum_{k=1}^{n} E_{k}\right) \\
& =\sum_{n=1}^{\infty} n \int_{E}^{E_{0}} d E_{1} f_{n}\left(E_{1}\right) P_{n-1}\left(E_{0}-E_{1}\right)=\int_{E}^{E_{0}} d E_{1}\left\{f_{1, e}\left(E_{1}\right)+f_{1, r}\left(E_{1}\right)\right\}+S_{t s}\left(E_{0}, E\right),
\end{aligned}
$$

where

$$
\begin{aligned}
\frac{d \delta_{t s}}{d E}= & \sum_{n=1}^{\infty} \frac{n P_{n, t s}\left(E_{0}\right)\left(E / \epsilon_{n}\right)^{p_{n}-1} e^{-E / \epsilon_{n}}}{\epsilon_{n} \Gamma\left(p_{n}\right) P\left(n p_{n}, E_{0} / \epsilon_{n}\right)} \\
& \times P\left[(n-1) p_{n},\left(E_{0}-E\right) / \epsilon_{n}\right],
\end{aligned}
$$

where we have used Eq. (A5). Here $P(z, x)$ is the normalized incomplete gamma function, which satisfies $P(0, x)=1$ (see Appendix A).

In the limit when $E_{0} \gg E, \epsilon_{n}$ we obtain the simplified formula

$$
\frac{d \delta_{t s}}{d E}=\sum_{n=1}^{\infty} n P_{n, t s}\left(E_{0}\right) \frac{\left(E / \epsilon_{n}\right)^{p_{n}-1} e^{-E / \epsilon_{n}}}{\epsilon_{n} \Gamma\left(p_{n}\right)}
$$

which shows that each component peaks at $E=\left(p_{n}-\right.$ 1) $\epsilon_{n}$. Furthermore, if all the $p_{n}$ 's and all the $\epsilon_{n}$ 's are equal, we obtain the simple result

$$
\frac{d \delta_{t s}}{d E}=\delta_{t s}\left(E_{0}\right) \frac{(E / \epsilon)^{p-1} e^{-E / \epsilon}}{\epsilon \Gamma(p)}
$$

where $p=p_{n}$ and $\epsilon=\epsilon_{n}$. This result shows that the function $f_{n}(E)$ can be identified with the energy spectrum if the energy $E_{0}$ is sufficiently high and the $f_{n}$ 's are independent of $n$. Note that, in this case, $d \delta_{t s} / d E$ peaks

The cumulative spectrum can be similarly computed.

where the true-secondary component is given by

$$
S_{t s}\left(E_{0}, E\right)=\sum_{n=1}^{\infty} \frac{n P_{n, t s}\left(E_{0}\right)}{\Gamma\left(p_{n}\right) P\left(n p_{n}, E_{0} / \epsilon_{n}\right)} \int_{E / \epsilon_{n}}^{E_{0} / \epsilon_{n}} d y y^{p_{n}-1} e^{-y} P\left[(n-1) p_{n}, E_{0} / \epsilon_{n}-y\right] .
$$

It is easily seen that this expression yields (52) upon taking $\partial / \partial E$ and reversing the sign. Unfortunately, it does not appear possible to express it in terms of conventional special functions.

\section{COMPUTATIONAL ALGORITHM}

The model defined in Secs. II and III is implemented, in practical simulations $[9,10]$, by the following algorithm:

(1) When an electron strikes the vacuum chamber wall, record its incident energy $E_{0}$ and the collision point $(x, y, z)$; compute the angle $\theta_{0}$ between the incident electron and the normal to the surface at $(x, y, z)$.
(2) Compute $\delta_{e}\left(E_{0}, \theta_{0}\right)$ according to Eqs. (25)-(46) and (47a), $\delta_{r}\left(E_{0}, \theta_{0}\right)$ according to Eqs. (28)-(46), (47a), and (47b), and $\delta_{t s}\left(E_{0}, \theta_{0}\right)$ according to Eqs. (31)-(48).

(3) Compute $P_{n}$ for $n=0,1, \ldots, M$ according to the model chosen ${ }^{3}$ in Sec. IIID2.

(4) Generate a random integer $n \in[0, M]$ with probability distribution $\left\{P_{n}\right\}$; this is the actual number of secondaries generated.

(5) If $n=0$, delete the incident electron from computer memory.

\footnotetext{
${ }^{3}$ In the computer calculation, we limit the number of secondary electrons generated in any given event to a maximum value $M$, even for the case of the Poisson distribution; typically we set $M=10$.
} 
(6) If $n=1$, generate the electron energy $E \in\left[0, E_{0}\right]$ with probability density $f_{1, e}(E)+f_{1, r}(E)+f_{1, t s}(E)$ (see Appendix 1).

(7) If $n \geq 2$, generate the energies $E_{k} \in\left[0, E_{0}\right], k=$ $1, \ldots, n$, with probability density $f_{n, t s}(E)$ such that $\sum_{k=1}^{n} E_{k} \leq E_{0}$ (see Appendix 2).

(8) Generate $n$ independent polar angles $\theta_{k} \in[0, \pi / 2]$ with probability density $\cos ^{\alpha} \theta$, and $n$ independent azimuthal angles $\phi_{k} \in[0,2 \pi]$ with uniform probability density. These are the emission angles of the secondary electrons relative to the local coordinate system that is centered at the collision point and whose " $z$ " axis is along the inward normal to the chamber surface.

(9) From the knowledge of the vacuum chamber geometry, the location $(x, y, z)$ and $(E, \theta, \phi)_{k}$ compute the momentum $\left(p_{x}, p_{y}, p_{z}\right)_{k}$ for each of the $n$ emitted electrons.

(10) Continue with the next incident electron at step (1).

\section{FITS TO EXPERIMENTAL DATA}

For illustration purposes, we have carried out a fit to experimental data at normal incidence for stainless steel and copper. The stainless steel data were obtained from a sample of SLAC standard 304 rolled sheet chemically etched and passivated but not conditioned $[21,29]$. For our fits we have used measured values of $\delta\left(E_{0}\right)$ in the range $0 \leq E_{0} \leq 1100 \mathrm{eV}$ (Fig. 4), and of $d \delta / d E$ at $E_{0}=$ $300 \mathrm{eV}$ (Fig. 5) and $1100 \mathrm{eV}$ (not shown). The copper data was obtained at CERN from a chemically cleaned but not in situ vacuum-baked sample [24]. We have used for our fits data for $\delta\left(E_{0}\right)$ in the range $0 \leq E_{0} \leq 1000 \mathrm{eV}$ (Fig. 6), and for $d \delta / d E$ at $E_{0}=10,30$, and $300 \mathrm{eV}$ (Fig. 7).

When fitting the backscattered peak, as seen in Figs. 5 and 7 , we deliberately tried to double the height of the experimentally measured peak. The reason is that our

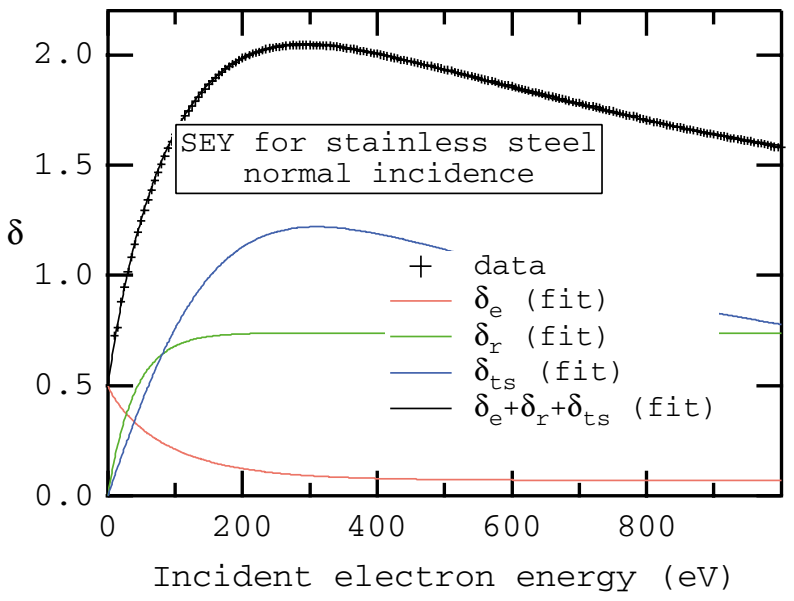

FIG. 4. (Color) The SEY for stainless steel for SLAC standard 304 rolled sheet, chemically etched and passivated but not conditioned. The parameters of the fit are listed in Table I. Data courtesy of R. Kirby.

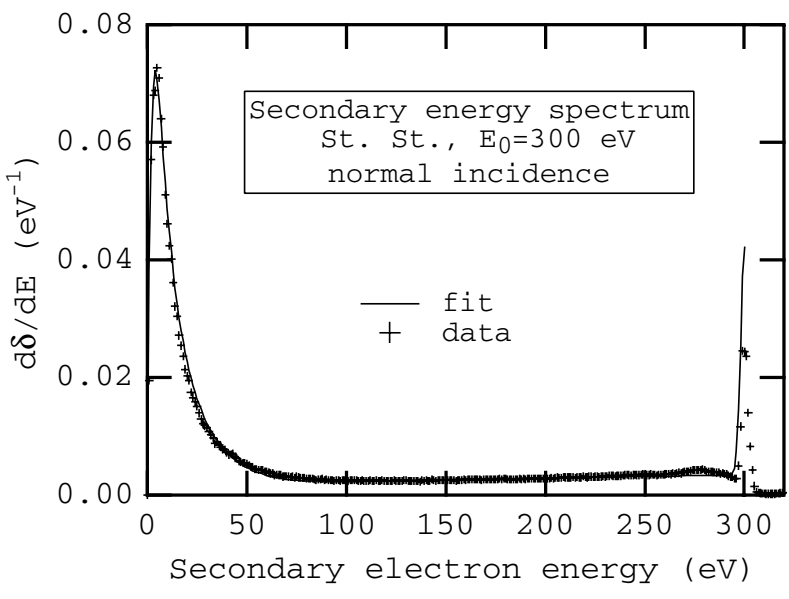

FIG. 5. The emitted-energy spectrum for stainless steel at $300 \mathrm{eV}$ incident energy and normal incidence, for SLAC standard 304 rolled sheet, chemically etched and passivated but not conditioned. Data courtesy of R. Kirby.

fitting curve for $d \delta / d E$ stops exactly at the maximum of the peak [viz. Eq. (26)], hence by doubling the height we ensure that the area under the peak, which we believe to be a better measure of $\delta_{e}$, matches the measured value.

We have obtained the parameters pertaining to incident-angle dependence $e_{1}, e_{2}, r_{1}, r_{2}$, and $t_{1}, \ldots, t_{4}$, appearing in Eqs. (47) and (48), from other sets of data for TiN-coated aluminum and for uncoated aluminum $[21,29]$. The parameter $\alpha$, which controls the angular distribution of the emitted electrons, is not determined by the above data. However, the value $\alpha=1$ is well supported by data for the true-secondary component elsewhere [17], hence we have adopted it. The angular distribution of the backscattered and rediffused components, however, differs substantially from a $\cos \theta$ distribution [17]. Nevertheless, we have set $\alpha=1$ for these components as well as for the sake of expediency, as we have

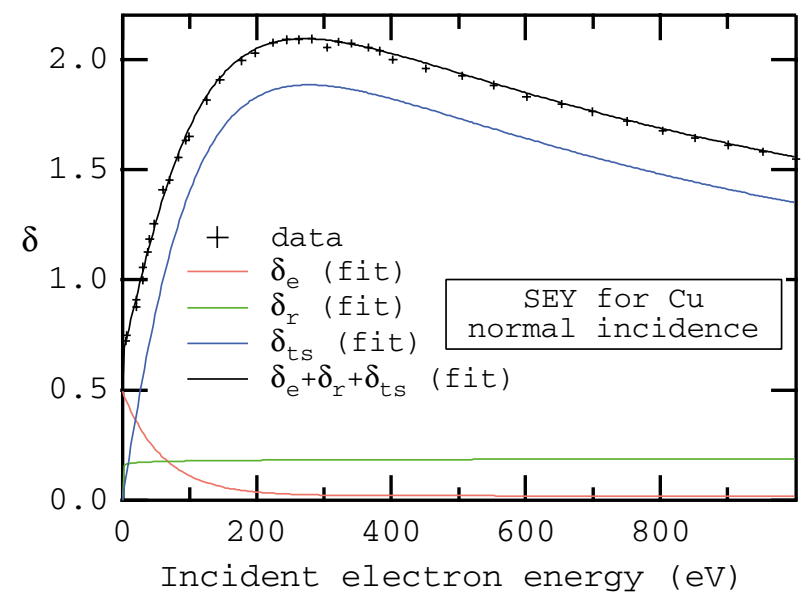

FIG. 6. (Color) The SEY for copper. The parameters of the fit are listed in Table I. Data courtesy of N. Hilleret for chemically cleaned but not in situ vacuum-baked samples. 

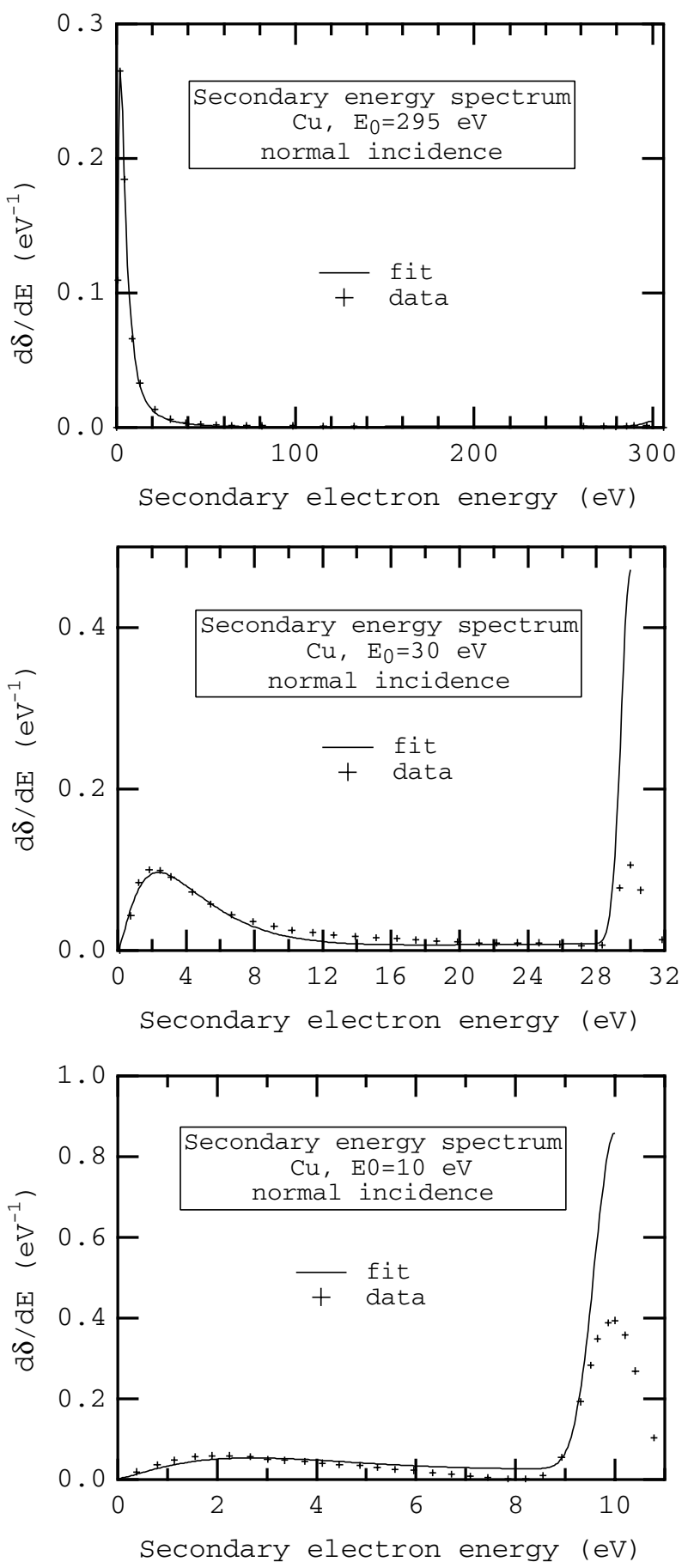

FIG. 7. The emitted-energy spectrum for copper at 295, 30, and $10 \mathrm{eV}$ incident energy and normal incidence on chemically cleaned but not in situ vacuum-baked samples. Data courtesy of N. Hilleret.

noted that electron-cloud simulations do not appear to be very sensitive to the exact value of $\alpha$. An improved parametrization of the angular dependence of these components is clearly necessary, and we intend to carry it out in the future [30]. Tables I and II list the values of our fitting parameters.
TABLE I. Main parameters of the model.

\begin{tabular}{|c|c|c|}
\hline & Copper & Stainless steel \\
\hline \multirow{3}{*}{$\alpha$} & $\begin{array}{l}\text { Emitted angular spectrum } \\
\text { (Sec. IIC } 1)\end{array}$ & \\
\hline & 1 & 1 \\
\hline & $\begin{array}{l}\text { Backscattered electrons } \\
\text { (Sec. IIIB) }\end{array}$ & \\
\hline$P_{1, e}(\infty)$ & 0.02 & 0.07 \\
\hline$\hat{P}_{1, e}$ & 0.496 & 0.5 \\
\hline$\hat{E}_{e}(\mathrm{eV})$ & 0 & 0 \\
\hline$W(\mathrm{eV})$ & 60.86 & 100 \\
\hline$p$ & 1 & 0.9 \\
\hline$\sigma_{e}(\mathrm{eV})$ & 2 & 1.9 \\
\hline$e_{1}$ & 0.26 & 0.26 \\
\hline \multirow[t]{2}{*}{$e_{2}$} & 2 & 2 \\
\hline & $\begin{array}{l}\text { Rediffused electrons } \\
\quad \text { (Sec. IIIC) }\end{array}$ & \\
\hline$P_{1, r}(\infty)$ & 0.2 & 0.74 \\
\hline$E_{r}(\mathrm{eV})$ & 0.041 & 40 \\
\hline$r$ & 0.104 & 1 \\
\hline$q$ & 0.5 & 0.4 \\
\hline$r_{1}$ & 0.26 & 0.26 \\
\hline \multirow[t]{2}{*}{$r_{2}$} & 2 & 2 \\
\hline & $\begin{array}{c}\text { True-secondary electrons } \\
\text { (Sec. IIID) }\end{array}$ & \\
\hline$\hat{\boldsymbol{\delta}}_{t s}$ & 1.8848 & 1.22 \\
\hline$\hat{E}_{t s}(\mathrm{eV})$ & 276.8 & 310 \\
\hline$s$ & 1.54 & 1.813 \\
\hline$t_{1}$ & 0.66 & 0.66 \\
\hline$t_{2}$ & 0.8 & 0.8 \\
\hline$t_{3}$ & 0.7 & 0.7 \\
\hline \multirow[t]{2}{*}{$t_{4}$} & 1 & 1 \\
\hline & Total SEY ${ }^{a}$ & \\
\hline$\hat{E}_{t}(\mathrm{eV})$ & 271 & 292 \\
\hline$\hat{\boldsymbol{\delta}}_{t}$ & 2.1 & 2.05 \\
\hline
\end{tabular}

$\overline{\text { aNote that } \hat{E}_{t} \simeq \hat{E}_{t s} \text { and } \hat{\delta}_{t} \simeq \hat{\delta}_{t s}+P_{1, e}(\infty)+P_{1, r}(\infty) \text { provided }}$ that $\hat{E}_{t s} \gg \hat{E}_{e}, E_{r}$.

The value of the SEY at low incident energy deserves special attention. At primary energies below a few eV no secondary electrons are created. In our model, only the backscattered component contributes to $\delta(0)$, with $\delta(0) \propto$ $\hat{P}_{1, e}$. The value $\hat{P}_{1, e} \simeq 0.5$ (see Table I), which we obtained by smoothly extrapolating the above-mentioned data down to $E_{0}=0$ for both the copper and stainless steel samples, is also supported (for stainless steel) by comparing recent observations for the decay time of the electron cloud in the Proton Storage Ring [31,32] with simulation results based on the SEY model described here. This parameter, which is sensitive to the potential field at the metal surface, may be a function of the surface composition, state of conditioning, and $\delta_{\max }$. Previous analytic work [18,33,34] yields estimates for $\delta(0) \sim$ 0.07 or lower for the case of smooth crystalline surfaces. 
TABLE II. Additional model parameters for the true-secondary component.

\begin{tabular}{ccc}
\hline \hline & Copper & Stainless steel \\
\hline$p_{n}$ & $2.5,3.3,2.5,2.5,2.8,1.3,1.5,1.5,1.5,1.5$ & $1.6,2,1.8,4.7,1.8,2.4,1.8,1.8,2.3,1.8$ \\
$\epsilon_{n}(\mathrm{eV})$ & $1.5,1.75,1,3.75,8.5,11.5,2.5,3,2.5,3$ & $3.9,6.2,13,8.8,6.25,2.25,9.2,5.3,17.8,10$ \\
\hline \hline
\end{tabular}

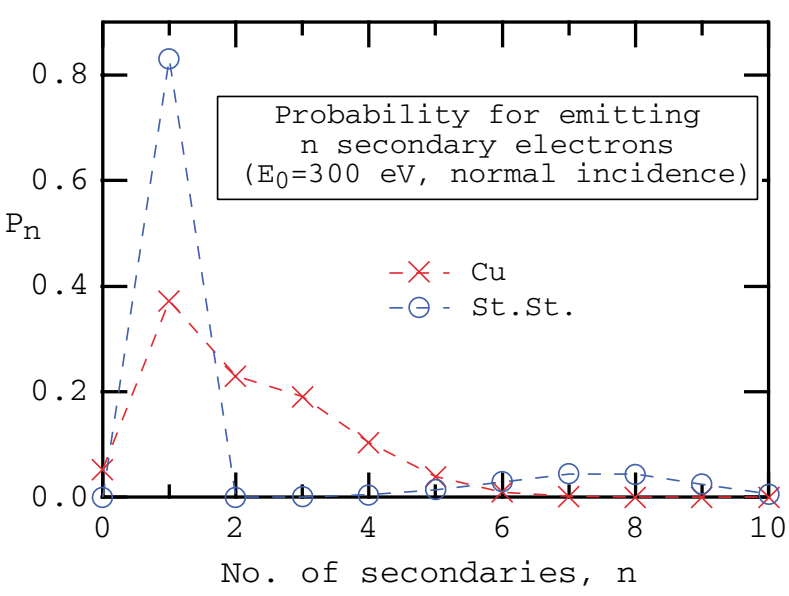

FIG. 8. (Color) The probability for emitting $n$ secondary electrons when a $300-\mathrm{eV}$ electron strikes a surface at normal incidence, computed according to Eqs. (43)-(46) using the fit parameters listed in Table $I$ assuming a binomial form [Eq. (46)] for $P_{n, t s}^{\prime}$ with $M=10$. The large relative value of $P_{1}$ for stainless steel is due to the large value of $\delta_{e}+\delta_{r}$.

It is interesting to note that Eq. (39) implies that $\delta_{t s}^{\prime}$ becomes large when $\delta_{e}+\delta_{r}$ approaches unity, hence so does $\langle n\rangle$ (unless, of course, $\delta_{t s}$ is correspondingly small). Therefore, as implied by Eqs. (43) combined with either (45) or (46), the distribution of the $P_{n}$ 's will be bimodal, with a prominent peak at $n=1$ and a secondary peak at some larger value of $n$. An example of this situation is shown in Fig. 8 for the case of stainless steel at $300 \mathrm{eV}$ incident energy. Since, for the binomial distribution choice, $\delta_{t s}^{\prime}$ must be $<M$, this kind of consideration must be used to determine an acceptable lower value for $M$. For the Poisson distribution choice it is desirable to truncate it at $n=M \gg \delta_{t s}^{\prime}$, otherwise the truncated distribution may lead to significant numerical errors.

\section{DISCUSSION AND CONCLUSIONS}

We have presented a mathematically self-consistent phenomenological probabilistic model for the secondaryemission process. The basic mathematical building block of the model is the most differential probability $\mathbb{P}_{n}$ for the emission of $n$ electrons when an incident electron strikes a surface at a given energy and angle. The inputs to the model are the three components of the SEY and the secondary energy spectrum. From these four phenomenological quantities we have constructed an event-by-event probabilistic description of the secondary-emission pro- cess suitable for multiparticle simulations of the ECE. The mathematical self-consistency of the model ensures that these four input quantities are recovered upon performing a statistical average over a large number of events.

The main assumptions in the model are the following: (1) The $n$ secondary electrons are generated instantaneously when a primary electron hits a surface. ${ }^{4}$ (2) The backscattered and rediffused electrons are generated only in single-electron events $(n=1)$. (3) The true-secondary electrons are generated in events for arbitrary $n \geq 1$ with a distribution in $n$ whose mean is the true-secondary component of the SEY. (4) The emission energies of the $n$ secondary electrons generated in any given event follow an almost uncorrelated distribution such that (i) the energy of any given emitted electron does not exceed the incident energy $E_{0}$, and (ii) the aggregate energy of the emitted electrons does not exceed $E_{0}$ either. (5) The emission angles of the $n$ secondary electrons generated in any given event are (i) fully uncorrelated, (ii) independent of the incident energy and angle, and (iii) uncorrelated with the emission energies.

The model contains a fair number of adjustable parameters, more than can be unambiguously determined from the data at present. Consequently, many of the parameters cannot be uniquely pinned down, although some are more robustly constrained by the data than others. The parameter set we have presented in Tables I and II gives a good overall representation of the data for the particular samples we have analyzed, but it is possible that other data sets may be equally acceptable. The parameters $\hat{\boldsymbol{\delta}}_{t s}, \hat{E}_{t s}, s, \sigma_{e}, \hat{P}_{1, e}, P_{1, e}(\infty)$, and $P_{1, r}(\infty)$ are robustly determined by the data. For copper, the $\epsilon_{n}$ 's and $p_{n}$ 's are reasonably well pinned down for $n \leqslant 4$, and for stainless steel for $4 \leq n \leq 8$.

As mentioned in the Introduction, we had previously used, incorrectly, the formula for the jet energy spectrum (Appendix B) to extract the model parameters from the data [9]. Although the parameters so extracted are somewhat different from those presented in Sec. VI, the overall features of the model, and the electron-cloud simulations obtained from it, remain qualitatively unchanged.

\footnotetext{
${ }^{4}$ This assumption is well justified, since the time lag of secondary emission [14] is estimated to be $10^{-13}-10^{-14} \mathrm{~s}$, i.e., much shorter than any time scale relevant to the electron-cloud effect.
} 
The model allows for certain flexibility without relinquishing its self-consistency. Specifically, the model accepts almost arbitrary parametrizations for the three components of the SEY (backscattered, rediffused, and true secondary) as functions of incident energy and angle. The energy spectrum, on the other hand, is more restricted: while its backscattered and rediffused components are almost arbitrary, its true-secondary component is constrained to be of the form [viz., Eq. (52)]

$$
\sum_{n} C_{n}\left(E, E_{0}\right) E^{p_{n}-1} e^{-E / \epsilon_{n}},
$$

where $E$ is the secondary energy. Here the parameters $p_{n}$ and $\epsilon_{n}$ are freely adjustable (as long as they are positive) independently of the SEY. The functions $C_{n}\left(E, E_{0}\right)$, however, are determined by the true-secondary yield and by the $p_{n}$ 's and $\epsilon_{n}$ 's. The form (57) is determined by that of the $f_{n}$ 's, Eq. (33), which was, in turn, chosen because the data for the spectrum look qualitatively like $E^{p-1} e^{-E / \epsilon}$, and also because it allows many of the integrals in our analysis to be carried out analytically. It is possible that other parametrizations may give a better representation of details of present or future data. In a future publication we intend to analyze the sensitivity of electron-cloud simulations against various parameter dependencies in our model [30].

As more measurements of the SEY and the energy spectrum become available, we expect to steadily improve our model and to better pin down the parameters. As an example, we have recently found that the fits to the copper data in Sec. VI require a dependence of $\sigma_{e}$ on the incident energy $E_{0}$. The three data sets in Fig. 7 require a monotonically increasing dependence of $\sigma_{e}$ on $E_{0}$ which we have parametrized in the form

$$
\sigma_{e}\left(E_{0}\right)=\sigma_{e 1}-1.88+2.5 \tanh \left[\left(E_{0}-150\right) / 100\right] \text {, }
$$

where all the numerical constants are in units of $\mathrm{eV}$, and $\sigma_{e 1}$ is specified in Table I as $\sigma_{e}$. Obviously this fit is far from unique, and we do not know how it extrapolates to $E_{0}>300 \mathrm{eV}$. Furthermore, we do not know the detection resolution of the apparatus used to measure the elastic peaks in Fig. 7. It is reasonable to assume that the resolution varies with $E_{0}$, so this effect would have to be unfolded from the data in order to obtain a more faithful dependence of $\sigma_{e}$ on $E_{0}$. As another example of further refinements, we have noted a dependence of $s$ on $\theta_{0}$ for aluminum samples [21], of the form

$$
s=1.43-0.0033 \theta_{0},
$$

where $\theta_{0}$ is in degrees. However, owing to the limited character of the fits presented here, we cannot disentangle this dependence from others in our model, hence we have assumed, for the purposes of this article, that $s$ is independent of $\theta_{0}$, as specified in Table I. As an example of potential future improvements, we find it reasonable to expect that the parameter $\alpha$, which controls the shape of the angular distribution of the emitted electrons, may well depend on both $n$ (the number of electrons emitted in the event) and on $E_{0}$. Our model can be readily augmented to accommodate any of the above-mentioned dependencies without disturbing its self-consistency.

\section{ACKNOWLEDGMENTS}

We are indebted to R. Kirby and N. Hilleret for kindly providing us much data and for many discussions. We are grateful to M. Blaskiewicz, I. Collins, R. Cimino, D. Trbojevic, and F. Zimmermann for valuable discussions. We are grateful to NERSC for supercomputer support. This work was supported by the U.S. DOE under Contract No. DE-AC03-76SF00098 and by the SNS project (ORNL).

\section{APPENDIX A: MATHEMATICAL DETAILS}

Here we provide a list of useful multidimensional integrals used in the calculations above. The basic integral

$$
I_{n}(x, p)=\frac{1}{\Gamma^{n}(p)} \int_{0}^{\infty} d x_{1} \cdots d x_{n}\left(x_{1} \cdots x_{n}\right)^{p-1} \delta\left(x-x_{1}-\cdots-x_{n}\right)=\theta(x) \frac{x^{n p-1}}{\Gamma(n p)}, \quad p>0
$$

is easily proved by induction in $n$. Note that $I_{n}$ obeys the recursion formula

$$
I_{n}(x, p)=\frac{1}{\Gamma(p)} \int_{0}^{\infty} d y y^{p-1} I_{n-1}(x-y, p),
$$

where consistency demands the definition $I_{0}(x, p)=\delta(x)$.

Integrating $I_{n}(x, p)$ with respect to $x$ yields

$$
\int_{0}^{x} d x I_{n}(x, p)=\frac{1}{\Gamma^{n}(p)} \int_{0}^{\infty} d x_{1} \cdots d x_{n}\left(x_{1} \cdots x_{n}\right)^{p-1} \theta\left(x-x_{1}-\cdots-x_{n}\right)=\theta(x) \frac{x^{n p}}{\Gamma(n p+1)}, \quad p>0 .
$$

Another related integral is

$$
F_{n}(x, p)=\frac{1}{\Gamma^{n}(p)} \int_{0}^{\infty} d x_{1} \cdots d x_{n}\left(x_{1} \cdots x_{n}\right)^{p-1} e^{-\left(x_{1}+\cdots+x_{n}\right)} \delta\left(x-x_{1}-\cdots-x_{n}\right)=\theta(x) \frac{x^{n p-1} e^{-x}}{\Gamma(n p)}, \quad p>0
$$

which is obtained in a straightforward way from (A1). Integrating this with respect to $x$ yields 


$$
G_{n}(x, p)=\frac{1}{\Gamma^{n}(p)} \int_{0}^{\infty} d x_{1} \cdots d x_{n}\left(x_{1} \cdots x_{n}\right)^{p-1} e^{-\left(x_{1}+\cdots+x_{n}\right)} \theta\left(x-x_{1}-\cdots-x_{n}\right)=\theta(x) P(n p, x), \quad x, p>0,
$$

where $P(z, x)$ is the normalized incomplete gamma function, defined, in general, by

$$
P(z, x)=\frac{\gamma(z, x)}{\Gamma(z)}=\frac{1}{\Gamma(z)} \int_{0}^{x} d t t^{z-1} e^{-t},
$$

where $x \geq 0$ and $\operatorname{Re} z>0$, and where $\gamma(z, x)$ is the ordinary incomplete gamma function [28]. Note that $G_{n}$ obeys the recursion formula

$$
G_{n}(x, p)=\frac{1}{\Gamma(p)} \int_{0}^{\infty} d y y^{p-1} e^{-y} G_{n-1}(x-y, p)
$$

where consistency demands the definition $G_{0}(x, p)=$ $\theta(x)$. In terms of the $P$ function, this recursion formula implies

$$
P(n p, x)=\frac{1}{\Gamma(p)} \int_{0}^{x} d y y^{p-1} e^{-y} P[(n-1) p, x-y],
$$

where $n \geq 1, x \geq 0$, and $p>0$ [in the right-hand side of this formula we must use $P(0, x)=\lim _{z \rightarrow 0^{+}} P(z, x)=1$ for the case $n=1]$.

\section{APPENDIX B: THE “JET” ENERGY SPECTRUM}

A spectrum that has been confused [9] with $d \delta / d E$ is the "jet energy spectrum," defined to be the secondary energy spectrum that would be obtained by a detector able to measure the number of emitted electrons $n$ and their aggregate energy $E=E_{1}+\cdots+E_{n}$ on an eventby-event basis. This spectrum is analogous to the differential jet cross sections of certain final states obtained in collisions of high energy particles. For the secondaryemission process, this type of measurement is in principle possible but probably impractical as it requires event-byevent measurements.

In the context of our model, the spectrum that such a detector would measure is given by the expression

$$
\left(\frac{d \delta}{d E}\right)_{\text {jet }}=\sum_{n=1}^{\infty} n \int(d E)_{n}(d \Omega)_{n} \mathbb{P}_{n} \delta\left(E-\sum_{k=1}^{n} E_{k}\right)
$$

which should be compared with Eq. (11). This spectrum obeys the sum rule

$$
\int_{0}^{E_{0}} d E\left(\frac{d \delta}{d E}\right)_{\text {jet }}=\sum_{n=1}^{\infty} n P_{n}=\delta,
$$

namely the same as $d \delta / d E$. Assuming the model described in Sec. III for the three components of the SEY and using Eq. (A4) we obtain

$$
\left(\frac{d \delta}{d E}\right)_{\text {jet }}=f_{1, e}+f_{1, r}+\left(\frac{d \delta_{t s}}{d E}\right)_{\text {jet }}
$$

where

$$
\left(\frac{d \delta_{t s}}{d E}\right)_{\mathrm{jet}}=\sum_{n=1}^{\infty} n P_{n, t s}\left(E_{0}\right) \frac{\left(E / \epsilon_{n}\right)^{n p_{n}-1} e^{-E / \epsilon_{n}}}{\epsilon_{n} \gamma\left(n p_{n}, E_{0} / \epsilon_{n}\right)},
$$

and where $\gamma(z, x)$ is the ordinary incomplete gamma function, defined in Eq. (A6). This result should be compared with Eq. (52) for the emitted-energy spectrum. Note that $\left(d \delta_{t s} / d E\right)_{\text {jet }}$ is broader than $d \delta_{t s} / d E$, as each of its components peaks at $E=\left(n p_{n}-1\right) \epsilon_{n}$ in the former rather than $E=\left(p_{n}-1\right) \epsilon_{n}$ in the latter. Therefore, the measurement of $\left(d \delta_{t s} / d E\right)_{\text {jet }}$, if it were possible, would provide knowledge about $P_{n, t s}$ for higher values of $n$ than $d \delta_{t s} / d E$, which would add valuable information about the secondary-emission process.

\section{APPENDIX C: FULLY UNCORRELATED MODEL FOR $\mathbb{P}_{n}$}

In this simplified model we assume that $d P_{n} /(d E)_{n}$ is given by

$$
\frac{d P_{n}}{(d E)_{n}}=\prod_{k=1}^{n} f_{n}\left(E_{k}\right) \theta\left(E_{k}\right)
$$

The absence of correlation among the $E_{k}$ 's embodied in Eq. (C1), as opposed to Eq. (17), simplifies the calculation because it allows one to deal with the emitted electrons independently of each other. On the other hand, the fact that the energies $E_{k}$ are not subject to any constraint other than being positive implies a nonzero probability that energy is not conserved because there is nothing to prevent the sum of the $E_{k}$ 's, or, indeed, any individual $E_{k}$, from exceeding $E_{0}$. Equation (C1) represents an approximation relative to (17) that is valid when $E_{0}$ is large compared with the typical values for the emitted energies, a situation that is sometimes realized in practice.

If the function $f_{n}(E)$ is assumed to be of the form (33) we obtain

$$
F_{n}^{n}=\frac{P_{n, t s}\left(E_{0}\right)}{\left[\epsilon_{n}^{p_{n}} \Gamma\left(p_{n}\right)\right]^{n}}
$$

which is the high $E_{0}$ limit of Eq. (34), as it should be. To derive this result we used

$$
\int_{0}^{\infty} d E f_{n}(E)=P_{n}^{1 / n}\left(E_{0}\right)
$$

which follows from Eq. (8). In this fully uncorrelated model the emitted-energy spectrum is given by 


$$
\begin{aligned}
\frac{d \delta_{t s}}{d E} & =\sum_{n=1}^{\infty} n f_{n, t s}(E) P_{n, t s}^{1-1 / n}\left(E_{0}\right) \\
& =\sum_{n=1}^{\infty} n P_{n, t s}\left(E_{0}\right) \frac{\left(E / \epsilon_{n}\right)^{p_{n}-1} e^{-E / \epsilon_{n}}}{\epsilon_{n} \Gamma\left(p_{n}\right)} .
\end{aligned}
$$

Similarly, the jet energy spectrum is

$$
\left(\frac{d \delta_{t s}}{d E}\right)_{\text {jet }}=\sum_{n=1}^{\infty} n P_{n, t s}\left(E_{0}\right) \frac{\left(E / \epsilon_{n}\right)^{n p_{n}-1} e^{-E / \epsilon_{n}}}{\epsilon_{n} \Gamma\left(n p_{n}\right)} .
$$

Expressions (C4) and (C5) are the limiting forms of (52) and (B4) when $E_{0} \gg E, \epsilon_{n}$, as should be expected according to the discussion above.

\section{APPENDIX D: MONTE CARLO GENERATION OF SECONDARY ELECTRONS}

\section{THE CASE $\boldsymbol{n}=1$}

The energy $E$ of an emitted electron in an event in which only one electron is generated is determined probabilistically according to the distribution density

$$
\frac{d N}{d E} \propto f_{1}(E)=f_{1, e}(E)+f_{1, r}(E)+f_{1, t s}(E) .
$$

If the standard accept-reject method is applied to the function $f_{1}(E)$, it is easy to encounter conditions for which this technique is very inefficient owing to the prominent peaks of $f_{1}(E)$ at $E \gtrsim 0$ and $E \lesssim E_{0}$, particularly when $E_{0}>100 \mathrm{eV}$. Since the inverse of the cumulative distribution for $f_{1}(E)$ cannot be found in closed form in terms of conventional functions, it is not practical to use the inversion technique either. However, it is easy to find the inverse of the cumulative distribution of each of the three functions $f_{1, e}(E), f_{1, r}(E)$, and $f_{1, t s}(E)$ separately, in which case an efficient technique (the so-called "composition rule") does exist [35]. ${ }^{5}$

The problem is restated as follows: generate a random number $E \in\left[0, E_{0}\right]$ with distribution density

$$
\rho(E)=a_{e} \rho_{e}(E)+a_{r} \rho_{r}(E)+a_{t s} \rho_{t s}(E),
$$

where the $\rho$ 's are probability densities with unit normalization, defined by

$$
\begin{aligned}
\rho_{e}(E) & =f_{1, e}(E) / \delta_{e}\left(E_{0}\right), \\
\rho_{r}(E) & =f_{1, r}(E) / \delta_{r}\left(E_{0}\right), \\
\rho_{t s}(E) & =f_{1, t s}(E) / P_{1, t s}\left(E_{0}\right),
\end{aligned}
$$

and the weights $a_{i}$ 's satisfy $a_{i}>0$ and $a_{e}+a_{r}+a_{t s}=1$. Referring to Sec. III, these weights are given by

\footnotetext{
${ }^{5}$ We are indebted to M. Blaskiewicz for bringing this technique to our attention.
}

$$
\begin{aligned}
a_{e} & =\delta_{e}\left(E_{0}\right) / \delta_{1}\left(E_{0}\right), \\
a_{r} & =\delta_{r}\left(E_{0}\right) / \delta_{1}\left(E_{0}\right), \\
a_{t s} & =P_{1, t s}\left(E_{0}\right) / \delta_{1}\left(E_{0}\right),
\end{aligned}
$$

where $\delta_{1}\left(E_{0}\right) \equiv \delta_{e}\left(E_{0}\right)+\delta_{r}\left(E_{0}\right)+P_{1, t s}\left(E_{0}\right)$. The algorithm to generate $E$ is, then, the following:

(1) Generate a random number $u$ uniformly distributed in $[0,1]$.

(2) If $0 \leq u<a_{e}$, generate $E$ with probability density $\rho_{e}(E)$, i.e., $E=E_{0}-\sigma_{e}|g|$, where $g$ is a Gaussian random number with zero mean and unit standard deviation (reject it if $E<0$ ).

(3) If $a_{e} \leq u<a_{e}+a_{r}$, generate $E$ with probability density $\rho_{r}(E)$, i.e., $E=E_{0} u_{1}^{1 /(1+q)}$, where $u_{1}$ is another random number uniformly distributed in $[0,1]$.

(4) If $a_{e}+a_{r} \leq u<1$, generate $E$ with probability density $\rho_{t s}(E)$, i.e., $E=\epsilon_{1} P^{-1}\left(p_{1}, u_{2} P_{0}\right)$, where $P^{-1}\left(p_{1}, x\right)$ is the functional inverse (in $\left.x\right)$ of $P\left(p_{1}, x\right), u_{2}$ is another random number uniformly distributed in $[0,1]$, and $P_{0}=P\left(p_{1}, E_{0} / \epsilon_{1}\right)$. Here $P(p, x)$ is the normalized incomplete gamma function, Eq. (A6).

This algorithm has the added benefit that it identifies the generated electron as backscattered, rediffused, or true secondary, hence it allows the use of distinct emitted-angle distributions, in better agreement with the phenomenology [17].

\section{THE CASE $n \geq 2$}

The determination of the energies $E_{1}, \ldots, E_{n}$ of the true-secondary electrons emitted in an event in which $n$ electrons are generated is formally equivalent to the following mathematical problem: stochastically generate an $n$-dimensional vector $\mathbf{x}=\left(x_{1}, x_{2}, \ldots, x_{n}\right)$ with probability density

$$
\frac{d N}{d^{n} \mathbf{x}} \propto \theta\left(x_{0}-x_{1}-\cdots-x_{n}\right) \prod_{k=1}^{n} x_{k}^{p-1} e^{-x_{k}}
$$

subject to $x_{k} \geq 0$. In the above expression the components of $\mathbf{x}$ are the normalized energies, $x_{k}=E_{k} / \epsilon_{n}$, the power $p$ is what we called $p_{n}$ in the main body of this article, and $x_{0}=E_{0} / \epsilon_{n}$. These parameters must satisfy the conditions $x_{0} \geq 0$ and $p>0$.

If it were not for the constraint $x_{1}+\cdots+x_{n} \leq x_{0}$, the problem would factorize into $n$ elementary onedimensional weighted random number generations. The first step to factorize the distribution density is to define an auxiliary vector $\mathbf{y}$ via $x_{k}=y_{k}^{2}$. In order to preserve the one-to-one correspondence between $\mathbf{x}$ and $\mathbf{y}$, we require that $y_{k} \geq 0$. With this change of variables, the problem reduces to the stochastic generation of a vector $\mathbf{y}$ in the first "quadrant" with probability density

$$
\frac{d N}{d^{n} \mathbf{y}} \propto \theta\left(y_{0}-y\right) e^{-y^{2}} \prod_{k=1}^{n} y_{k}^{2 p-1},
$$


where $y=|\mathbf{y}|$ and $y_{0}=x_{0}^{1 / 2}$. The next step consists of going over to $n$-dimensional spherical coordinates for $\mathbf{y}$, namely,

$$
\left.\begin{array}{c}
y_{1}=y \cos \theta_{1}, \\
y_{2}=y \sin \theta_{1} \cos \theta_{2}, \\
y_{3}=y \sin \theta_{1} \sin \theta_{2} \cos \theta_{3}, \\
\vdots \\
y_{n-1}=y \sin \theta_{1} \sin \theta_{2} \cdots \cos \theta_{n-1}, \\
y_{n}=y \sin \theta_{1} \sin \theta_{2} \cdots \sin \theta_{n-1} .
\end{array}\right\}
$$

In the general case, when $\mathbf{y}$ is allowed to range over all space, the range for the angles is $0 \leq \theta_{k} \leq \pi$ for $k=$ $1, \ldots, n-2$, and $0 \leq \theta_{k} \leq 2 \pi$ for $k=n-1$. In our particular case, however, the restriction $y_{k} \geq 0$ implies that $0 \leq \theta_{k} \leq \pi / 2$ for all $k=1, \ldots, n-1$. Using the volume element

$$
d^{n} \mathbf{y}=y^{n-1} d y \prod_{k=1}^{n-1}\left(\sin \theta_{k}\right)^{n-k-1} d \theta_{k}
$$

we obtain

$$
d N \propto \theta\left(y_{0}-y\right) y^{2 n p-1} e^{-y^{2}} d y \prod_{k=1}^{n-1}\left(\sin \theta_{k}\right)^{2 \mu-1}\left(\cos \theta_{k}\right)^{2 \nu-1} d \theta_{k},
$$

where $\mu=p(n-k)$ and $\nu=p$. This distribution density is of the desired fully factorized form.

The angle $\theta_{k}$ is distributed with the probability density

$$
\frac{d N}{d \theta_{k}} \propto\left(\sin \theta_{k}\right)^{2 \mu-1}\left(\cos \theta_{k}\right)^{2 \nu-1},
$$

hence, with the change of variables $t=\sin ^{2} \theta_{k}$, we obtain

$$
\frac{d N}{d t} \propto t^{\mu-1}(1-t)^{\nu-1}, \quad 0 \leq t \leq 1,
$$

whose cumulative distribution is the normalized incomplete beta function,

$$
\hat{\beta}(x, \mu, \nu)=\frac{\Gamma(\mu+\nu)}{\Gamma(\mu) \Gamma(\nu)} \int_{0}^{x} d t t^{\mu-1}(1-t)^{\nu-1},
$$

hence the angles $\theta_{k}$ are stochastically generated by the formula

$$
\theta_{k}=\arcsin \sqrt{\hat{\beta}^{-1}\left(u_{k}, \mu, \nu\right)},
$$

where $\hat{\beta}^{-1}(x, \mu, \nu)$ is the functional inverse (in $x$ ) of $\hat{\beta}(x, \mu, \nu)$, the $u_{k}$ 's are independent random numbers uniformly distributed in $[0,1]$, and the arcsin function is restricted to the interval $[0, \pi / 2]$.

The variable $y$ is distributed with the probability density

$$
\frac{d N}{d y} \propto \theta\left(y_{0}-y\right) y^{2 n p-1} e^{-y^{2}} .
$$

By making the change of variables $x=y^{2}$ this yields

$$
\frac{d N}{d x} \propto \theta\left(x_{0}-x\right) x^{n p-1} e^{-x},
$$

where $x_{0}$ was previously defined. In this case the cumulative distribution is the normalized incomplete gamma function $P(n p, x)$, Eq. (A6), with $x$ restricted to the range $0 \leq x \leq x_{0}$, hence the variable $y$ is stochastically generated by the formula

$$
y=\sqrt{P^{-1}\left(n p, u P_{0}\right)},
$$

where $P^{-1}(n p, x)$ is the functional inverse (in $x$ ) of
$P(n p, x), u$ is a random number uniformly distributed in $[0,1]$, and $P_{0}=P\left(n p, x_{0}\right)$.

To summarize, the algorithm for generating the energies $E_{k}$ is the following:

(1) Compute $x_{0}=E_{0} / \epsilon_{n}$ and $P_{0}=P\left(n p, x_{0}\right)$.

(2) Generate $n-1$ independent random numbers $u_{k}, k=1, \ldots, n-1$, uniformly distributed in $[0,1]$, and compute the angles $\theta_{k}$ according to Eq. (D13).

(3) Generate one more random number $u$ uniformly distributed in $[0,1]$ and compute $y$ according to Eq. (D16).

(4) Compute the vector $\mathbf{y}$ according to Eq. (D7).

(5) Compute the energies using $E_{k}=\epsilon_{n} x_{k}=\epsilon_{n} y_{k}^{2}$ for $k=1, \ldots, n$.

The only potential difficulty to this algorithm is the need to evaluate the functions $\hat{\beta}^{-1}(x, \mu, \nu)$ and $P^{-1}(n p, x)$. However, computer libraries for statistical analysis typically have them available.

It may be convenient, for checking the validity of the numerical calculation, to obtain the one-dimensional projection of the distribution (D5). Using the integrals in Appendix A we obtain

$$
\frac{d N}{d x_{1}}=\frac{x_{1}^{p-1} e^{-x_{1}} P\left((n-1) p, x_{0}-x_{1}\right)}{\Gamma(p) P\left(n p, x_{0}\right)}, \quad 0 \leq x_{1} \leq x_{0},
$$

where we have chosen the normalization

$$
\int_{0}^{x_{0}} d x_{1} \frac{d N}{d x_{1}}=1
$$

(for the case $n=1$ we use $P(0, x)=1$ ).

\section{APPENDIX E: MONTE CARLO COMPUTATION OF THE SPECTRA}

Besides the analytic expressions (52) and (B4), the energy spectra $d \delta_{t s} / d E$ and $\left(d \delta_{t s} / d E\right)_{\text {jet }}$ can be computed using the Monte Carlo technique by stochastically simulating the generation of secondary electrons and appropriately binning their energies. The main benefit of this Monte Carlo technique is to validate the computational 
algorithm for the secondary-emission process described in Sec. V, which is used in our main ECE simulation code.

The technique consists of obtaining a secondary energy histogram in $E$. For this purpose we fix all model parameters and then use the algorithm in Sec. V for a large number $N_{0}$ of incident electrons, all of which have the same incident energy $E_{0}$ and incident angle $\theta_{0}$. We define the histogram by dividing the energy interval $\left[0, E_{0}\right]$ into a certain number $B$ of bins of size $\Delta E=$ $E_{0} / B$. Let $N_{i}$ be the number of events in bin $i$, where the index $i=1,2, \ldots, B$ labels the bin corresponding to the energy interval $[(i-1) \Delta E, i \Delta E]$.

To compute $d \delta_{t s} / d E$ we generate secondary electrons for each incident (primary) electron according to the algorithm in Sec. V, except that we add the following instructions ${ }^{6}$ immediately following step (7):

(7a) For the $k$ th secondary electron, compute the bin number $i$ corresponding to its energy $E_{k}$.

(7b) Increment $N_{i}$ by 1 .

For the computation of $\left(d \delta_{t s} / d E\right)_{\text {jet }}$ the appropriate steps that replace (7a) and (7b) above are the following:

(7a) Compute the total secondary energy $E_{\text {tot }}=E_{1}+$ $E_{2}+\cdots+E_{n}$.

(7b) Compute the bin number $i$ corresponding to $E_{\text {tot }}$.

(7c) Increment $N_{i}$ by $n$.

The histograms are then normalized by multiplying $N_{i}$ by $\delta\left(E_{0}\right) /\left(N_{s} \Delta E\right)$, where $N_{s}$ is the total number of secondaries generated by the $N_{0}$ primary electrons, and $\delta\left(E_{0}\right)$ is the SEY.

The Monte Carlo calculation also yields in a straightforward manner the probabilities $P_{n}$ by simply tallying the events with $n$ emitted electrons, then dividing by the total number $N_{0}$ of incident electrons. This computation can be used as a check of the validity of the technique, since the $P_{n}$ 's thus obtained must agree, within statistical errors, with the input values given by Eqs. (35) or (43), depending on the model chosen.

The above procedures (except for the trivial normalization of the histograms) are implemented by a section of FORTRAN code seen in Listing 1.

Listing 1 contains iPn_hist, idde_hist, and iddejet_hist as the histograms for $P_{n}, d \delta / d E$, and $(d \delta / d E)_{\text {jet }}$, respectively, nprim is the number $N_{0}$ of primary electrons, desec is the energy interval size $\Delta E$, nstot is a counter that tallies the total number $N_{s}$ of secondary electrons, and the rest of the variables are fairly obvious. The main ingredient, of course, is the event generator subroutine secelec(E0,theta0,nsec,energ,ang) that creates secondary electrons for a given incident electron. This subroutine embodies steps (1)-(7) of the algorithm described in Sec. V. It takes as inputs the incident electron energy $E_{0}$ (variable E0) and the incident angle $\theta_{0}$ (variable theta 0 ),

\footnotetext{
${ }^{6}$ For the purposes of obtaining the energy spectra, steps 8 and 9 are skipped.
}

Listing 1. Computation of $P_{n}, d \delta / d E$, and $(d \delta / d E)_{\text {jet }}$.

do $1 \mathrm{np}=1$,nprim

call secelec(E0,theta0,nsec,energ,ang)

iPn_hist (nsec) $=$ iPn_hist $($ nsec $)+1$

if $(\mathrm{nsec}==0)$ go to 1

nstot $=$ nstot + nsec

Etot $=0$

do $n s=1, n s e c$

$\mathrm{En}=\mathrm{energ}(\mathrm{ns})$

Etot=Etot+En

ien $=$ En $/ \mathrm{desec}+1$

idde_hist (ien)=idde_hist (ien)+1

end do

ien=Etot $/ \mathrm{desec}+1$

iddejet_hist(ien)=iddejet_hist(ien)+nsec

1 continue

in addition to all the parameters listed in Table I. The outputs are the number of secondary electrons generated $n$ (variable nsec), their energies $E_{1}, E_{2}, \ldots, E_{n}$ contained in the one-dimensional array energ, and their polar and azimuthal angles contained in the two-dimensional array ang.

Figure 9 shows the two kinds of spectra, computed both by the analytic and Monte Carlo techniques, for a sample case in which $\delta_{e}=\delta_{r}=0$ and $\delta=\delta_{t s}=1.877$.

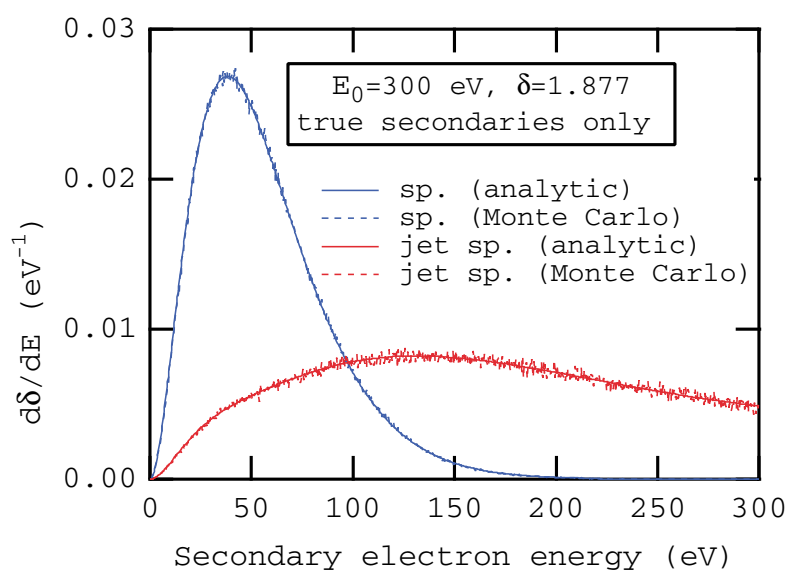

FIG. 9. (Color) The two kinds of secondary energy spectra for normal-incident electrons of energy $E_{0}=300 \mathrm{eV}$ for the parameter choices $p_{n}=3, \epsilon_{n}=20 \mathrm{eV}, \delta_{e}=\delta_{r}=0$, and $\delta_{t s}=$ $\delta=1.877$ assuming a Poisson distribution for $P_{n, t s}$ truncated at $n=10$. The two curves vanish identically beyond $E_{0}$ and are normalized so that the area under either of them in $0 \leq E \leq E_{0}$ equals $\delta$. The analytic curves are given by Eqs. (52) and (B4), respectively. For the Monte Carlo method we used $N_{0}=10^{6}$ incident electrons and we divided the energy interval $\left[0, E_{0}\right]$ into $10^{3}$ bins of width $\Delta E=0.3 \mathrm{eV}$. The values chosen for $\epsilon_{n}$ and $p_{n}$ are not meant to be realistic; we use them here for illustration purposes only. Note that $d \delta / d E$ peaks at $E \simeq(p-$ 1) $\epsilon=40 \mathrm{eV}$ in agreement with the discussion in Sec. IV, while $(d \delta / d E)_{\text {jet }}$ is much broader, as each of its components peaks at $E=(n p-1) \epsilon$, as discussed in Appendix B. 


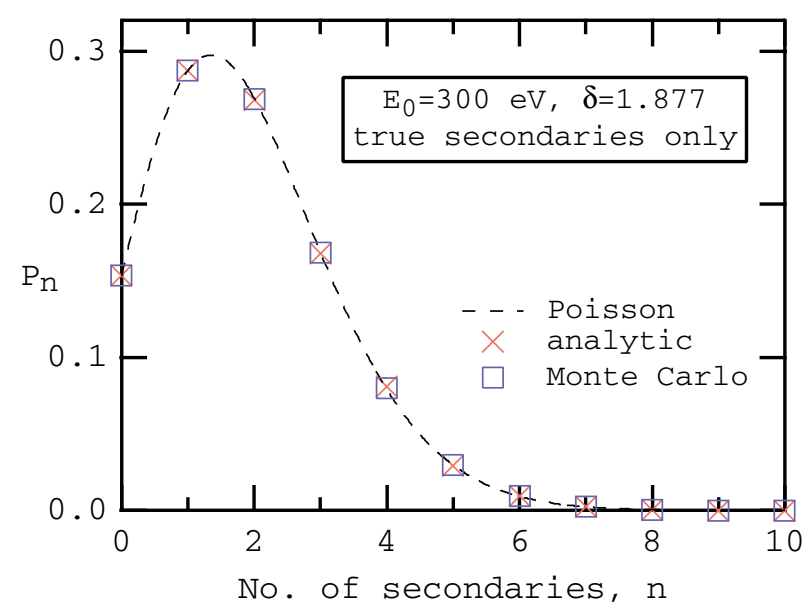

FIG. 10. (Color) The probability for emitting $n$ electrons, $P_{n}$, for the same conditions as in Fig. 9. The "Poisson" curve is Eq. (37), extended analytically to continuous values of $n$. The analytic results were obtained by numerically integrating $d \delta_{t s} / d E$, given by Eq. (52), over $E$ in the range $0 \leq E \leq E_{0}$ separately for each value of $n$. The Monte Carlo results were obtained concurrently with the energy spectra shown in Fig. 9, according to the computer algorithm described in Appendix E.

Figure 10 shows the result of the calculation of the emission probabilities $P_{n}$ for the same conditions for the energy spectrum. The agreement between the analytic and the Monte Carlo methods supports the validity of the subroutine secelec.

\section{APPENDIX F: ALTERNATIVE FITS FOR THE TRUE-SECONDARY YIELD}

Equation (32) is qualitatively similar to a more conventional form for the universal scaling function, namely [14],

$$
D(x)=a x^{1-s}\left(1-e^{-b x^{s}}\right),
$$

where $a, b$, and $s$ are constrained by the conditions $D(1)=1$ and $D^{\prime}(1)=0$, i.e.,

$$
a=\frac{1}{1-e^{-b}}, \quad s=\frac{1-e^{-b}}{1-(1+b) e^{-b}},
$$

which leaves only one independent parameter. Equation (F1) is obtained from the semiempirical theory of secondary emission $[17,23,25]$. In this framework the true-secondary yield is

$$
\delta_{t s} \propto \int_{0}^{R} d z f(z)\left(-\frac{d E}{d z}\right)
$$

where $d E / d z$ is the energy loss rate of the primary electron in the material, $f(z)$ is the probability that a secondary electron created at depth $z$ will make it to the surface, and $R$ is the range of the primary electron. The effects from scattering of the primary electron in the material are taken into account [14] by replacing $d E / d z$ by its effective value, $-E_{0} / R$. Assuming $f(z)=\exp (-z / \lambda)$, where $\lambda$ is the absorption length of a secondary electron in the material, Eq. (F3) yields

$$
\delta_{t s} \propto \frac{E_{0}}{R}\left(1-e^{-R / \lambda}\right) .
$$

Furthermore, assuming the validity of the energy-range relation $R \propto E_{0}^{s}$, (F4) yields (F1) upon trading off all proportionality constants for $\hat{E}$ and $\hat{\delta}$, and imposing the conditions $D(1)=1$ and $D^{\prime}(1)=0$. The parameter $s$ may be obtained from measurements of the range-energy relation for $\mathrm{Al}_{2} \mathrm{O}_{3}$, yielding $s=1.35$ [15], whence $a=1.11$ and $b=2.28$. Another example of an alternative form for $D(x)$ that differs from (F1) but satisfies, nevertheless, the range-energy relation, is found in Ref. [26]. Figure 11 shows Eqs. (32) and (F1) plotted for $s=1.35$.

It is straightforward to verify that our Eq. (32) can be obtained in the same way by simply assuming $f(z)=$ $(1+z / 2 \lambda)^{-2}$ instead of $\exp (-z / \lambda)$. Note that both (32) and (F1) have the same power-law behaviors at small and large $E_{0}$, namely,

$$
D(x) \propto \begin{cases}x & \text { as } x \rightarrow 0 \\ x^{1-s} & \text { as } x \rightarrow \infty\end{cases}
$$

on account of the validity of the energy-range relation. Since (32) provides a better fit to the SEY data than (F1) for the samples we have analyzed, it would appear that the secondary electron escape probability function $(1+$ $z / 2 \lambda)^{-2}$ describes the escape process better than $\exp (-z / \lambda)$. However, due to the limited energy range of our fits, and the number of other fitting parameters, we cannot draw this conclusion with certainty. Nevertheless, such a possibility might be worthy of further investigation.

For materials with smooth surfaces, the incident-angle dependence of $\hat{\delta}$ and $\hat{E}$ are often parametrized as

$$
\hat{\delta}\left(\theta_{0}\right)=\frac{\hat{\delta}(0)}{\left(\cos \theta_{0}\right)^{\alpha_{1}}}, \quad \hat{E}\left(\theta_{0}\right)=\frac{\hat{E}(0)}{\left(\cos \theta_{0}\right)^{\alpha_{2}}}
$$

where $\alpha_{1}$ and $\alpha_{2}$ are $\mathcal{O}(1)[16,26]$. The samples we have analyzed [21,27], however, correspond to amorphous

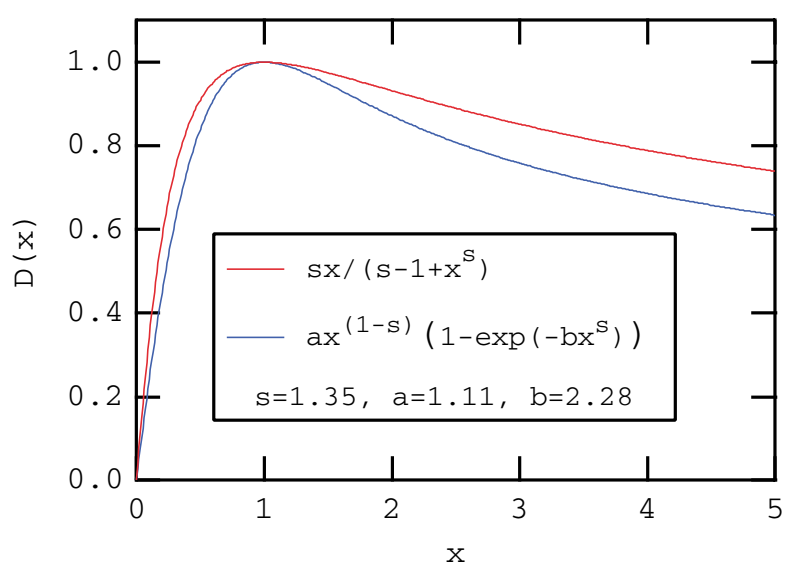

FIG. 11. (Color) The true-secondary emission yield scaling functions, Eqs. (32) and (F1), for $s=1.35$. 
materials with rough surfaces and we have found that the above power laws give a much too strong dependence on $\theta_{0}$. As described in Secs. IIIB, IIIC, and IIID, we have found that polynomial fits of the form $1+a_{1}(1-$ $\left.\cos ^{a 2} \theta_{0}\right)$ represent the data quite well for the range $0 \leq$ $\theta_{0} \lesssim 84^{\circ}$, particularly for $\hat{\delta}\left(\theta_{0}\right) / \hat{\delta}(0)$. However, a good alternative fit for this ratio, which is more conventional than the polynomial fit, is given by [17]

$$
\hat{\delta}\left(\theta_{0}\right)=\hat{\delta}(0) \exp \left[\gamma\left(1-\cos \theta_{0}\right)\right] .
$$

Actual fits to the data yield values for $\gamma$ in the range $\sim 0.2-0.7$ depending on the type of material, its state of conditioning, and the incident energy $E_{0}$ [21]. For the values in Table I, with $\left(a_{1}, a_{2}\right)$ representing any of the pairs $\left(e_{1}, e_{2}\right),\left(r_{1}, r_{2}\right),\left(t_{1}, t_{2}\right)$, or $\left(t_{3}, t_{4}\right)$, the following substitutions work adequately:

$$
\begin{aligned}
& \left(a_{1}, a_{2}\right)=(0.26,2.0) \rightarrow \gamma=0.33, \\
& \left(a_{1}, a_{2}\right)=(0.66,0.8) \rightarrow \gamma=0.49, \\
& \left(a_{1}, a_{2}\right)=(0.70,1.0) \rightarrow \gamma=0.56
\end{aligned}
$$

*Electronic address: mafurman@lbl.gov

†Electronic address: mpivi@lbl.gov

Address after January 1, 2003: SLAC, P.O. Box 20450, Stanford, CA 94309.

[1] M. Izawa, Y. Sato, and T. Toyomasu, Phys. Rev. Lett. 74, 5044-5047 (1995).

[2] K. Ohmi, Phys. Rev. Lett. 75, 1526-1529 (1995).

[3] O. Gröbner, in Proceedings of the 10th International Accelerator Conference, Serpukhov, 1977 (Protvino, 1977), p. 277.

[4] O. Gröbner, in Proceedings of the Particle Accelerator Conference (PAC97), Vancouver, 1997 (IEEE, Piscataway, NJ, 1997), p. 3589.

[5] Various contributions in Proceedings of the PAC 2001, Chicago, 2001, especially the surveys by F. Zimmermann, K. Harkay, and R. Macek, http:// pac2001.aps.anl.gov/

[6] Various contributions to the Proceedings of the TwoStream Instability Workshop, KEK, Tsukuba, 2001 (unpublished), http://conference.kek.jp/two-stream/.

[7] Various contributions to the Proceedings of the ECLOUD-02 Workshop [CERN Yellow Report No. CERN-2002-001 (to be published)], http:// slap.cern.ch/collective/ecloud02/.

[8] Various contributions to the Proceedings of the EPAC02, Paris, 2002 (to be published).

[9] M. A. Furman and G. R. Lambertson, in Proceedings of the International Workshop on Multibunch Instabilities in Future Electron and Positron Accelerators MBI97, KEK, Tsukuba, Japan, 1997 (KEK Report No. 97-17, edited by Y. H. Chin, 1997, p. 170).

[10] M. A. Furman, LBNL Report No. LBNL-41482/CBP Note 247, 1998; CERN LHC Project Report No. 180, 1998 (unpublished).

[11] F. Zimmermann, in Proceedings of the XI Chamonix LHC Workshop, 2001 (unpublished), pp. 144-159, http://
cern.web.cern.ch/CERN/Divisions/SL/

publications/ chamx2001/contents.html

[12] M. A. Furman and M.T.F. Pivi, in Proceedings of the EPAC02 Paris, 2002 (to be published); LBNL Report No. LBNL-50765, 2002 (unpublished).

[13] See, for example, K. Murata, T. Matsukawa, and R. Shimizu, Jpn. J. Appl. Phys. 10, 678-686 (1971); T. Koshikawa and R. Shimizu, J. Phys. D 7, 1303-1315 (1974); J. P. Ganachaud and M. Cailler, Surf. Sci. 83, 498-530 (1979).

[14] A. J. Dekker, in Secondary Electron Emission, edited by F. Seitz and D. Turnbull, Solid State Physics Vol. 6 (Academic Press, New York, 1958), pp. 251-315.

[15] J. R. Young, Phys. Rev. 103, 292-293 (1956).

[16] H. Seiler, J. Appl. Phys. 54, R1-R18 (1983).

[17] H. Bruining, Physics and Applications of Secondary Electron Emission (Pergamon Press, McGraw-Hill Book Co., New York, 1954).

[18] P. A. Redhead, J. P. Hobson, and E.V. Kornelsen, The Physical Basis of Ultrahigh Vacuum (Chapman and Hall, London, 1968), Chap. 4 (reprinted by the AIP in 1993 as part of the American Vacuum Society Classics series).

[19] G. Ertl and J. Kuppers, Low Energy Electrons and Surface Chemistry (Verlag Chemie, Weinheim, 1985).

[20] D. Briggs and M. P. Seah, Practical Surface Analysis: Auger and X-ray Photoelectron Spectroscopy (Wiley Interscience, Chichester, NY, 1990).

[21] R. E. Kirby and F. K. King, Nucl. Instrum. Methods Phys. Res., Sect. A 469, 1-12 (2001).

[22] J. B. Hudson, Surface Science: An Introduction (Butterworth-Heinemann, Stoneham, MA, 1992), Chap. 14.

[23] E. M. Baroody, Phys. Rev. 78, 780-787 (1950).

[24] V. Baglin, I. Collins, B. Henrist, N. Hilleret, and G. Vorlaufer, CERN LHC Project Report No. 472 (unpublished).

[25] R. G. Lye and A. J. Dekker, Phys. Rev. 107, 977-981 (1957).

[26] S. A. Schwarz, J. Appl. Phys.. 68, 2382 (1990).

[27] K. Kennedy, B. Harteneck, G. Millos, M. Benapfl, F. King, and R. Kirby, in Proceedings of the PAC 1997, Vancouver (Ref. [4]), p. 3568.

[28] M. Abramowitz and I. A. Stegun, Handbook of Mathematical Functions (Dover Publications Inc., Mineola, NY, 1970).

[29] R. Kirby (unpublished).

[30] M. A. Furman and M. Pivi (to be published).

[31] R. Macek, in Proceedings of the ECLOUD-02 Workshop, CERN Yellow Report No. CERN-2002-001, 2002 (to be published).

[32] M. A. Furman and M.T. F. Pivi, in Proceedings of the EPAC02, Paris, 2002 (to be published); LBNL Report No. LBNL-50765, 2002.

[33] C. Herring and M. H. Nichols, Rev. Mod. Phys. 21, 185 (1949).

[34] H. Heil and J. Hollweg, Phys. Rev. 164, 881-886 (1967); 164, 887-895 (1967).

[35] L. Devroye, Non-Uniform Random Variate Generation (Springer-Verlag, New York, 1986). 